\title{
Guiding transcranial brain stimulation by EEG/MEG to interact with ongoing brain activity and associated functions
}

Citation for published version (APA):

Thut, G., Bergmann, T. O., Fröhlich, F., Soekadar, S. R., Brittain, J-S., Valero-Cabré, A., Sack, A. T., Miniussi, C., Antal, A., Siebner, H. R., Ziemann, U., \& Herrmann, C. S. (2017). Guiding transcranial brain stimulation by EEG/MEG to interact with ongoing brain activity and associated functions: A position paper. Clinical Neurophysiology, 128(5), 843-857. https://doi.org/10.1016/j.clinph.2017.01.003

Document status and date:

Published: 01/05/2017

DOI:

10.1016/j.clinph.2017.01.003

Document Version:

Publisher's PDF, also known as Version of record

\section{Document license:}

Taverne

Please check the document version of this publication:

- A submitted manuscript is the version of the article upon submission and before peer-review. There can be important differences between the submitted version and the official published version of record.

People interested in the research are advised to contact the author for the final version of the publication, or visit the DOI to the publisher's website.

- The final author version and the galley proof are versions of the publication after peer review.

- The final published version features the final layout of the paper including the volume, issue and page numbers.

Link to publication

\footnotetext{
General rights rights.

- You may freely distribute the URL identifying the publication in the public portal. please follow below link for the End User Agreement:

www.umlib.nl/taverne-license

Take down policy

If you believe that this document breaches copyright please contact us at:

repository@maastrichtuniversity.nl

providing details and we will investigate your claim.
}

Copyright and moral rights for the publications made accessible in the public portal are retained by the authors and/or other copyright owners and it is a condition of accessing publications that users recognise and abide by the legal requirements associated with these

- Users may download and print one copy of any publication from the public portal for the purpose of private study or research.

- You may not further distribute the material or use it for any profit-making activity or commercial gain

If the publication is distributed under the terms of Article $25 \mathrm{fa}$ of the Dutch Copyright Act, indicated by the "Taverne" license above, 
Review

\title{
Guiding transcranial brain stimulation by EEG/MEG to interact with ongoing brain activity and associated functions: A position paper
}

\author{
Gregor Thut ${ }^{\mathrm{a}, *}$, Til Ole Bergmann ${ }^{\mathrm{b}}$, Flavio Fröhlich ${ }^{\mathrm{c}}$, Surjo R. Soekadar ${ }^{\mathrm{d}}$, John-Stuart Brittain ${ }^{\mathrm{e}}$, \\ Antoni Valero-Cabré ${ }^{\mathrm{f}}$, Alexander T. Sack ${ }^{\mathrm{g}}$, Carlo Miniussi ${ }^{\mathrm{h}}$, Andrea Antal ${ }^{\mathrm{i}}$, Hartwig Roman Siebner ${ }^{\mathrm{j}, \mathrm{k}}$, \\ Ulf Ziemann ${ }^{1}$, Christoph S. Herrmann ${ }^{\mathrm{m}}$
}

${ }^{a}$ Centre for Cognitive Neuroimaging, Institute of Neuroscience and Psychology, University of Glasgow, Glasgow, UK

${ }^{\mathrm{b}}$ Department of Neurology \&' Stroke, and Hertie Institute for Clinical Brain Research, Institute for Medical Psychology and Behavioral Neurobiology, University Hospital

Tübingen, Eberhard Karls University of Tübingen, Tübingen, Germany

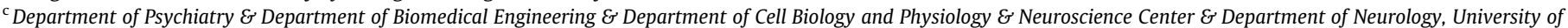
North Carolina at Chapel Hill, Chapel Hill, USA

${ }^{\mathrm{d}}$ Applied Neurotechnology Lab, Department of Psychiatry and Psychotherapy \& MEG Center, University Hospital of Tübingen, Tübingen, Germany

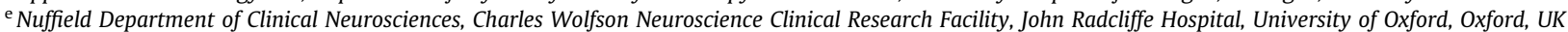

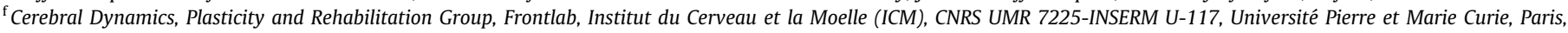
France

${ }^{\mathrm{g}}$ Department of Cognitive Neuroscience, Faculty of Psychology and Neuroscience, Maastricht University, Maastricht, The Netherlands

${ }^{\mathrm{h}}$ Center for Mind/Brain Sciences CIMeC University of Trento, Rovereto, Italy E Cognitive Neuroscience Section, IRCCS Centro San Giovanni di Dio Fatebenefratelli, Brescia, Italy

i Department of Clinical Neurophysiology, University Medical Center, Göttingen, Germany

${ }^{\mathrm{j}}$ Danish Research Centre for Magnetic Resonance, Centre for Functional and Diagnostic Imaging and Research, Copenhagen University Hospital Hvidovre, Hvidovre, Denmark

${ }^{\mathrm{k}}$ Department of Neurology, Copenhagen University Hospital Bispebjerg, Copenhagen, Denmark

${ }^{1}$ Department of Neurology E Stroke, and Hertie Institute for Clinical Brain Research, University Tübingen, Tübingen, Germany

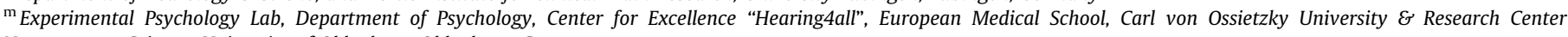
Neurosensory Science, University of Oldenburg, Oldenburg, Germany

\section{See Editorial, pages $839-840$}

\section{A R T I C L E I N F O}

\section{Article history:}

Accepted 8 January 2017

Available online 29 January 2017

\section{Keywords:}

Non-invasive transcranial brain stimulation (NTBS)

Electroencephalography

Magnetoencephalography

Brain oscillations

Temporally guided NTBS

\section{H I G H L I G H T S}

- We outline the opportunities of timing NTBS to ongoing brain activity for enhancing its efficacy.

- Emerging ideas emphasize brain oscillations as promising targets for interventions.

- This offers a principled framework for influencing the brain-behavior relationship by NTBS.

\section{A B S T R A C T}

Non-invasive transcranial brain stimulation (NTBS) techniques have a wide range of applications but also suffer from a number of limitations mainly related to poor specificity of intervention and variable effect size. These limitations motivated recent efforts to focus on the temporal dimension of NTBS with respect to the ongoing brain activity. Temporal patterns of ongoing neuronal activity, in particular brain oscillations and their fluctuations, can be traced with electro- or magnetoencephalography (EEG/MEG), to guide the timing as well as the stimulation settings of NTBS. These novel, online and offline EEG/MEG-guided NTBS-approaches are tailored to specifically interact with the underlying brain activity. Online EEG/MEG has been used to guide the timing of NTBS (i.e., when to stimulate): by taking into account instantaneous phase or power of oscillatory brain activity, NTBS can be aligned to fluctuations in excitability states. Moreover, offline EEG/MEG recordings prior to interventions can inform researchers and clinicians how to stimulate: by frequency-tuning NTBS to the oscillation of interest, intrinsic brain oscillations can be up- or down-regulated. In this paper, we provide an overview of existing approaches and ideas of EEG/

\footnotetext{
* Corresponding author at: 58 Hillhead Street, Glasgow G12 8QB, UK.

E-mail address: gregor.thut@glasgow.ac.uk (G. Thut).
} 
MEG-guided interventions, and their promises and caveats. We point out potential future lines of research to address challenges.

๑) 2017 International Federation of Clinical Neurophysiology. Published by Elsevier Ireland Ltd. All rights reserved.

\section{Contents}

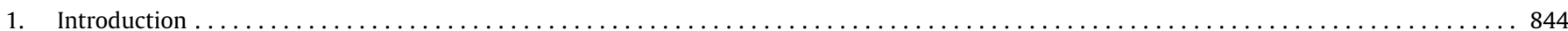

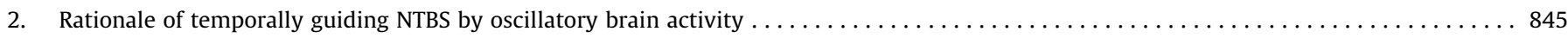

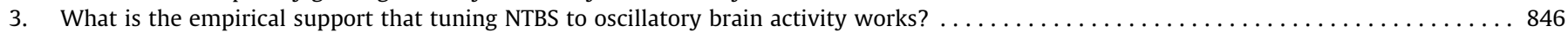

3.1. Enhancing NTBS efficacy by triggering TMS/TCS by instantaneous phase and/or power of underlying brain oscillations......... 846

3.2. Targeting brain activity and associated functions by frequency tuning of NTBS to underlying brain oscillations . . . . . . . . . 846

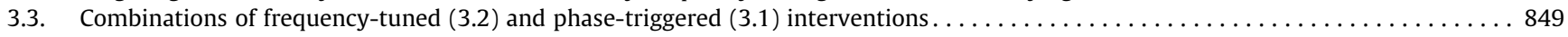

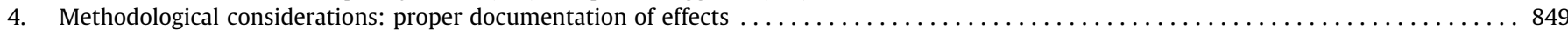

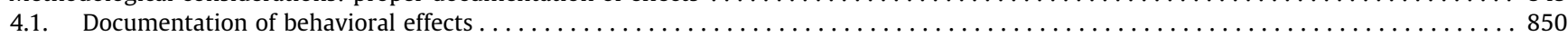

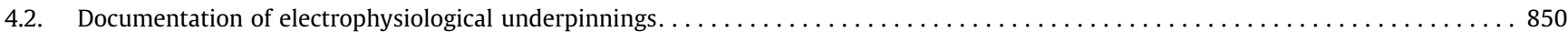

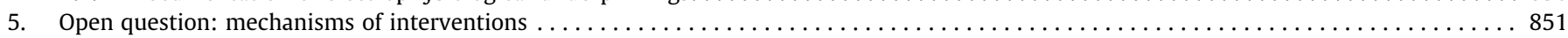

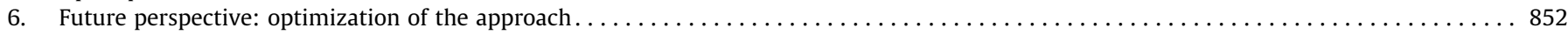

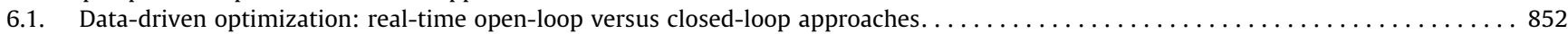

6.2. Model-driven optimization: prediction of effects with biologically plausible models and simulations $\ldots \ldots \ldots \ldots \ldots \ldots \ldots \ldots . \ldots \ldots 3$

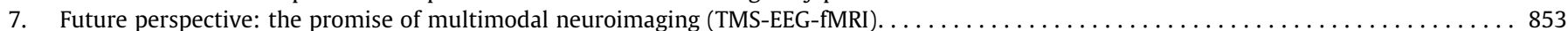

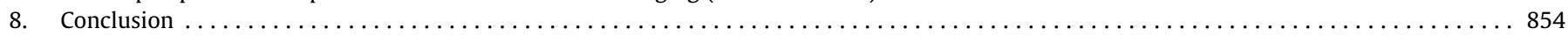

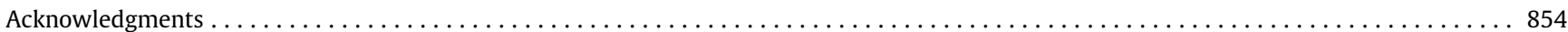

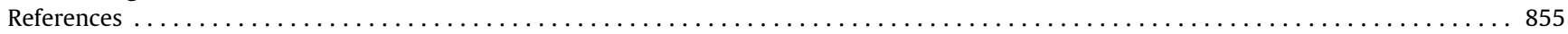

\section{Introduction}

Non-invasive transcranial brain stimulation (NTBS) of the human brain has gained notable popularity over the last three decades. Today, NTBS is widely used for experimental and clinical interventions in both healthy participants and patients. This is partially due to the development of transcranial magnetic stimulation (TMS) (Barker et al., 1985) and the re-discovery of transcranial current stimulation (TCS) protocols, including transcranial direct current stimulation (TDCS) (Nitsche and Paulus, 2000) or transcranial alternating current stimulation (TACS) as a variant (Antal et al., 2008), which are suitable in terms of ethics and safety for use in most individuals, and normally well tolerated by the participants (Rossi et al., 2009; Brunoni et al., 2011; Woods et al., 2016). Applications are multifold (Bergmann et al., 2016), ranging from studies on normal brain organization and reorganization (e.g. Fröhlich et al., 2015; Kuo and Nitsche, 2015; Prehn and Flöel, 2015) to biomarking (e.g. by recording TMS-evoked responses in electromyography or EEG, Bortoletto et al., 2015) and the development of plasticity inducing protocols (e.g. Liew et al., 2014; Karabanov et al., 2015; Wessel et al., 2015).

While NTBS has greatly advanced clinical neurophysiology and human neuroscience, some important limitations have become increasingly apparent over the years. One of the main limitations is a lack in understanding how NTBS interacts with brain activity at the neuronal level to give rise to behavioral effects, how these effects can most efficiently be optimized and how they may be influenced by intra- and inter-individual factors. Recent findings indicate a high inter- and intra-individual variability in NTBS outcomes across studies, despite the use of identical protocols, which depends on some known variables (such as age, gender, skull shape and structure, emotional and physiological state of participants before and during stimulation, see e.g. Li et al., 2015; Opitz et al., 2015; Ziemann and Siebner, 2015). However, these variables can only explain a small portion of the altogether considerable variability. Essentially, after-effects of commonly used NTBS protocols are challenging to interpret because of high intra- and inter- individual variability, small effect sizes at the group level, and limited reproducibility (Lally et al., 2013; Horvath et al., 2015). Currently, neurophysiologically grounded models of how NTBS interacts most efficiently with functionally relevant brain activity are largely lacking. A hypothesis-driven approach based on physiologically underpinned models is needed to guide the selection of NTBS parameters among the many to choose from.

The challenge is to understand how to target NTBS in order to efficiently interact with neuronal processes that underlie brain function such as perception, attention, memory, cognition or motor control. One important dimension of targeting is functional and structural neuroanatomy and an important tool is neuronavigation. However, the regional spatial specificity of NTBS is of concern. While the spatial resolution of TMS is relatively good (O'Shea and Walsh, 2007, Bolognini and Ro, 2010, but see Schmidt et al., 2015 for physical variability), TMS induced brain activity may spread from the area under the coil along neuronal connections to associated regions depending on the intensity of stimulation (see e.g. Siebner et al., 2000, 2001; Sack et al., 2007; Bestmann and Feredoes, 2013; Martin-Trias et al., 2016). Although the axonal and transsynaptic spread of excitation will be restricted by the anatomically predefined connectivity pattern, the spread of excitation limits inferences as to the anatomical origins and substrates of the associated behavioral effects. The spatial specificity of TCS is even more limited than TMS, due to low spatial resolution (Fertonani and Miniussi, 2016), although modeling of current distribution and new ideas for electrode montages (Miniussi et al., 2013; Klooster et al., 2016) suggest that spatial specificity may be increased to stimulate selective cortical structures in the future (Wang et al., 2014). Importantly, functional brain activity is not only defined by spatially distinct networks, but also by dynamic interactions within and across local and large-scale network components, reflected in brain oscillations across different frequency bands (Buzsaki and Draguhn, 2004). As a consequence, a recent line of research focused on the promise of adding a temporal to the spatial dimension of targeting, and considering brain oscillations as targets for intervention (e.g. Romei et al., 2016). 


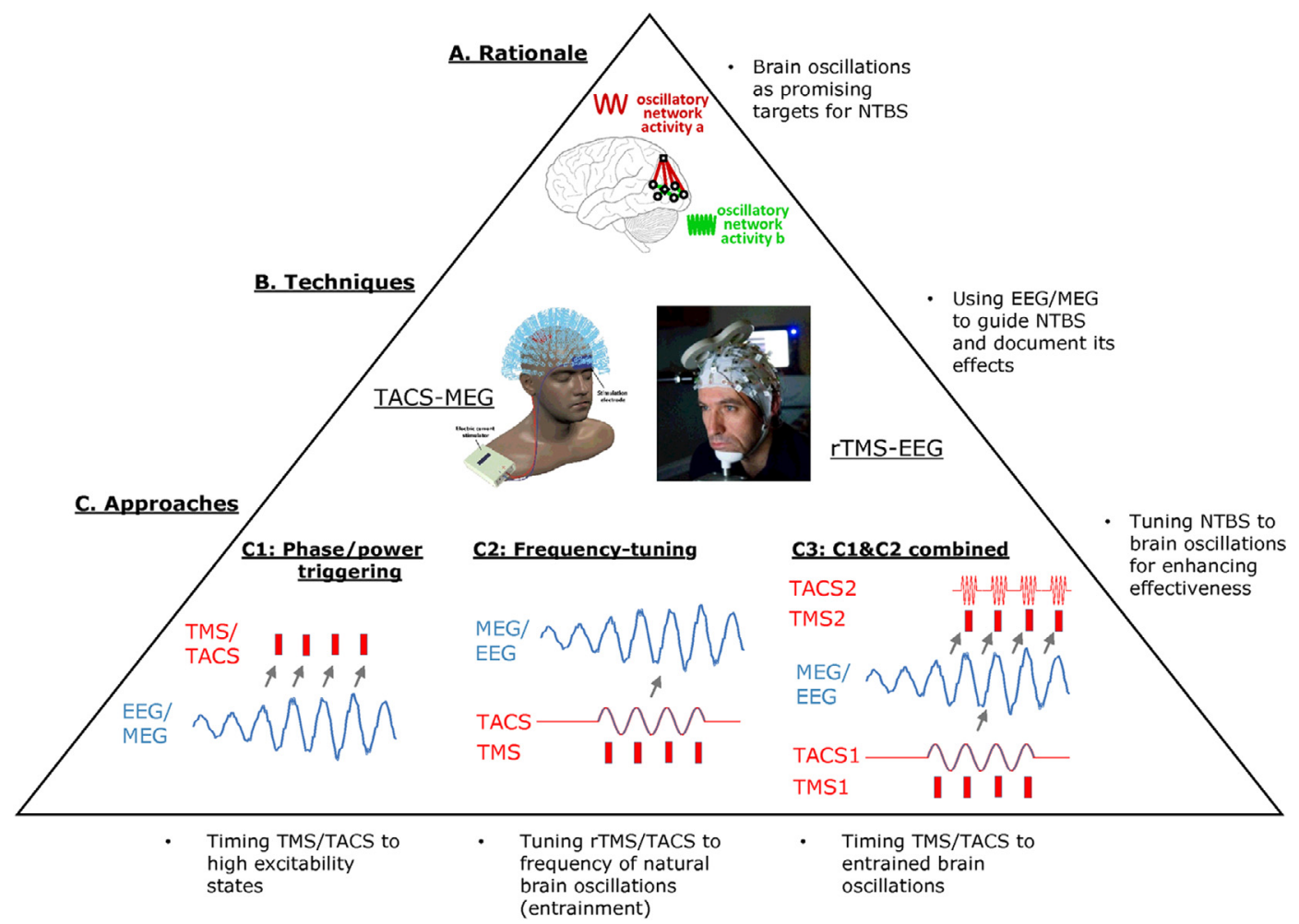

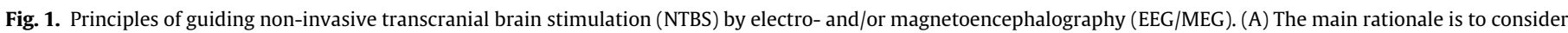

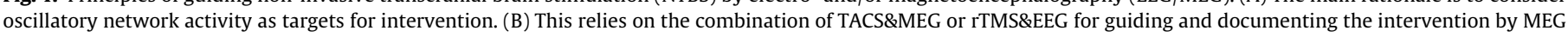

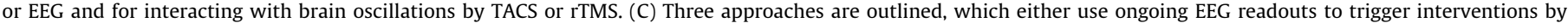

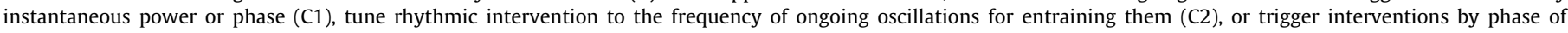
entrained oscillations (C3). See text for details and Figs. 2-4 for examples of each of these three approaches.

In this paper, we outline the opportunities afforded by considering the timing of NTBS intervention relative to ongoing brain activity as a window for enhancing its efficacy. In particular, we point out new ideas for tuning the timing of NTBS to ongoing brain oscillations that are based on recent advances in the understanding of the EEG/MEG signal, and illustrate these ideas with recent evidence from TMS-EEG and/or TCS-EEG/MEG studies. We outline the rationale and promise of this approach (Section 2), which covers new ideas about how to tailor TMS/TCS to promising brain signatures (or motifs) for interventions. We survey the growing body of evidence that this approach might work (Section 3), and consider practical issues on how to best document the effects (Section 4). We consider possible underlying mechanisms suggested by models and empirical data (Section 5), and outline important future lines of research on how to optimize the approach (Section 6). The latter includes the use of real-time interventions and the generation of biologically plausible models to guide the choices of stimulation parameters. Finally, we consider the promise of multimodal neuroimaging (TMS-EEG-fMRI) in future research (Section 7). Fig. 1 illustrates the main ideas of the approach.

\section{Rationale of temporally guiding NTBS by oscillatory brain activity}

Recent research combining EEG or MEG with TMS or TCS has revealed that oscillatory brain activity is a promising neural target for NTBS-based interventions to shape brain-behavior relationships.

In terms of their generation, most brain oscillations are reflecting network activity, as they are generated in specific local or large-scale neuronal networks (Buzsaki and Draguhn, 2004), although local pacemaker cells with an intrinsic rhythm do exist (e.g., in the thalamus), and depend on the current vigilance state as they are under strong control of brainstem neuromodulatory systems (Lee and Dan, 2012; Zagha and McCormick, 2014). In addition, most neuronal oscillations, such as those in the theta (4$8 \mathrm{~Hz})$, alpha $(8-12 \mathrm{~Hz})$, beta $(15-30 \mathrm{~Hz})$ and gamma bands $(>30 \mathrm{~Hz})$ rely to some degree on the phasic inhibitory activity of GABAergic interneurons (Wang, 2010), either expressed at a local level (as for gamma) or at a larger scale (such as for the thalamocortical alpha rhythm, see e.g. Lorincz et al., 2009). In contrast, the neocortical slow oscillation $(<1 \mathrm{~Hz})$ observed during nonrapid eye movement (NREM) sleep may merely result from spontaneous mini-EPSPs triggering persistent sodium currents (rather than from a pronounced GABAergic drive), which initiate a transient depolarization phase (the 'up-state') that turns into a subsequent hyperpolarization phase (the 'down-state') due to depolarization-activated potassium currents and synaptic depression (Bazhenov et al., 2002; Hill and Tononi, 2005). By means of its alternating 'down-states' of wide-spread neuronal silence and 'up-states' of increased neuronal firing, the slow oscillation is grouping faster activity such as sleep spindles $(12-15 \mathrm{~Hz})$ (Steriade et al., 1993; Steriade, 2006). Even slower, so called 'infra-slow' oscillations $(\sim 0.1 \mathrm{~Hz})$, modulate the amplitude of basically all faster oscillations during wakefulness through yet unknown (maybe phasic neuromodulatory) mechanisms (Monto et al., 2008).

Importantly, brain oscillations are associated with various different 'circuit motives' that are recurrent throughout the brain, serving comparable computational functions (Womelsdorf and Everling, 2015). Irrespective of the mechanism of generation, the interplay of excitatory and inhibitory neurons within those circuits usually results in periodic fluctuations of the excitation-inhibition balance (EIB) and eventually in variations in the neurons' 
membrane potential between states of relative de- and hyperpolarization (Buzsaki and Draguhn, 2004; Schroeder and Lakatos, 2009). The associated alternation between high and low excitability states at the level of a given neuron results in rhythmic gain modulation of both its synaptic input (with EPSPs more easily overcoming firing threshold) and its output (firing rate being modulated by the neuron's membrane potential) (e.g., Haegens et al., 2011). This rhythmic input/output gain modulation in turn underlies a series of higher order functional principles of neuronal oscillations, such as communication-through-coherence (Fries, 2005, 2015), hierarchical nesting as indexed by phase-power-coupling (Jensen and Colgin, 2007; Schroeder et al., 2008), phase precession and phase coding (Lisman, 2005; Schyns et al., 2011; Jensen et al., 2014), and gating by pulsed inhibition (Jensen and Mazaheri, 2010). Moreover, disinhibition, reflected in a transient deflection of the EIB towards relative excitation by release from inhibition, has been proposed as an important mechanism serving plastic processes at the network level (Letzkus et al., 2015; Cash et al., 2016). Together, these principles presumably provide a temporal framework as well as the basic computational building blocks of neuronal network interactions in a variety of sensorimotor and cognitive processes (Varela et al., 2001; VanRullen and Koch, 2003; Buzsaki and Draguhn, 2004; Schroeder and Lakatos, 2009).

When synchronized across sufficiently large populations of interconnected neurons, brain oscillations are observable in the local field potential (LFP) and ultimately also non-invasively in surface EEG and MEG, thereby reflecting instantaneous markers of neuronal network excitability (Buzsáki et al., 2012). Given that a neuron's current state of excitability is an essential factor modulating both the gating and communication of signals as well as the induction of synaptic plasticity within neuronal networks and given the proposed roles of brain oscillations in a variety of cognitive functions, both the oscillatory phase (reflecting current excitability) and amplitude (reflecting current degree of local neuronal synchronization) represent worthwhile targets for NTBS interventions (see also Thut et al., 2012). For instance, tuning NTBS to high excitability phases of oscillatory brain activity may enhance efficacy of NTBS as compared to when applied at random phases. Similarly, synchronizing or desynchronizing brain oscillations by frequency-tuning of electromagnetic stimulation to ongoing oscillations (e.g. by their entrainment) may offer the opportunity to intervene with brain activity and associated functions at a fundamental (mechanistic) level of network interactions.

\section{What is the empirical support that tuning NTBS to oscillatory brain activity works?}

Several ideas of how NTBS can interact with neuronal oscillations have emerged. The main distinction is between research on immediate and longer-lasting changes, respectively focusing on the effects during NTBS (resulting from direct neuronal excitation/inhibition and interaction with ongoing brain activity) or the after-effects (due to NTBS-induced longer-term changes in excitability or activity immediately following NTBS and beyond). These can be further subdivided in approaches that (1) trigger TMS/TCS by instantaneous oscillatory phase and/or power, (2) tune TMS/TCS to the natural frequency of the underlying oscillation versus (3) a combination of both, as is outlined below (and is schematically represented in Fig. 1C).

\subsection{Enhancing NTBS efficacy by triggering TMS/TCS by instantaneous phase and/or power of underlying brain oscillations}

The general idea is that the effectiveness of NTBS can be enhanced by timing NTBS to specific phase and/or power values of ongoing brain oscillations (Fig. 1C, left panel). There are indeed several examples of early EEG-TMS studies which demonstrated using post hoc trial sorting - a relationship between the effectiveness of a single TMS pulse and the power and phase of ongoing brain oscillations at the time of its delivery, as revealed by phase- and/or power-modulation of the amplitude of motor evoked potentials (MEPs) or phosphene induction to TMS over the motor or visual cortex, respectively. The size of the TMSevoked MEP scales with the power of ongoing sensorimotor $\mu$ rhythms $(8-15 \mathrm{~Hz})$ directly preceding the TMS pulse (e.g., Sauseng et al., 2009a; Schulz et al., 2014). Likewise, phosphene reports depend on the power of posterior alpha oscillations immediately preceding occipital TMS (Romei et al., 2008a,b). Moreover, MEPs and phosphene reports have been shown to vary, respectively, with the instantaneous phase of sensorimotor $\mu$-rhythms (Triesch et al., 2015) and posterior alpha oscillations (Dugue et al., 2011) at time of stimulation. Finally, using EEG-triggered TMS, Bergmann et al. (2012a) explicitly targeted up- and downstates of slow oscillation during NREM sleep. It was shown that motor cortical excitability during deep sleep fluctuates in a phase-dependent manner, with larger MEPs and TMS evoked potentials (TEPs) being evoked during slow oscillation EEG upstates and smaller MEPs/TEPs during slow oscillation downstates of the stimulated motor cortex, and the absolute voltage at the time of stimulation further predicting within-state MEP/TEP amplitude (illustrated in Fig. 2).

In addition, recent studies indicate that power and phase at time of TMS may not only influence the immediate effects of TMS, but also TMS after-effects. It has been suggested that by tuning TMS pulses of plasticity-inducing protocols to instantaneous periods of low versus high excitability, plasticity effects may be enhanced. For example, repeated stimulation into either the low or high excitability phase of the sensorimotor $\mu$-oscillation may induce LTP- and LTD-like motor cortical plasticity respectively (Triesch et al., 2015; Zrenner et al., 2015, 2016), in analogy to theta phase-specific plasticity demonstrated in the rodent hippocampus (Huerta and Lisman, 1993, 1995). The rationale behind this approach is to repetitively generate neuronal input, precisely timed to phases of high excitability/disinhibition, thus increasing the chances of TMS-induced postsynaptic firing, and by extension of spike timing dependent plasticity (STDP)-like processes to occur (Artola et al., 1990; Sjöström et al., 2001). In other words, the oscillatory amplitude and/or phase are used as target windows for plasticity inducing protocols. This requires the repeated, temporally precise targeting to a priori defined periods of high excitability in specific oscillatory frequency bands, which has now become feasible with real-time EEG-triggered TMS (see Section 6.1).

\subsection{Targeting brain activity and associated functions by frequency tuning of NTBS to underlying brain oscillations}

While the approach depicted above utilizes EEG data on instantaneous phase or power for triggering of TMS to enhance TMS efficacy, an alternative methodology aims at tuning rhythmic stimulation protocols (such as repetitive TMS/rTMS or transcranial alternating current stimulation/TACS) to the frequency of an ongoing brain oscillation (Fig. $1 \mathrm{C}$, middle panel). It has been shown that this protocol can result in phase-coupling (also termed entrainment) between oscillatory brain activity and the external electromagnetic stimulus, opening new ways to investigate (and modulate) the relationship between aspects of oscillatory brain activity (such as its phase and amplitude) and behavior.

In terms of immediate effects during NTBS, there are multiple examples that this approach may indeed lend itself for a targeted intervention into oscillatory brain activity through entrainment that can affect brain function and behavior, both using TMS or 
A. Design

single

pulseTMS

(hand area)

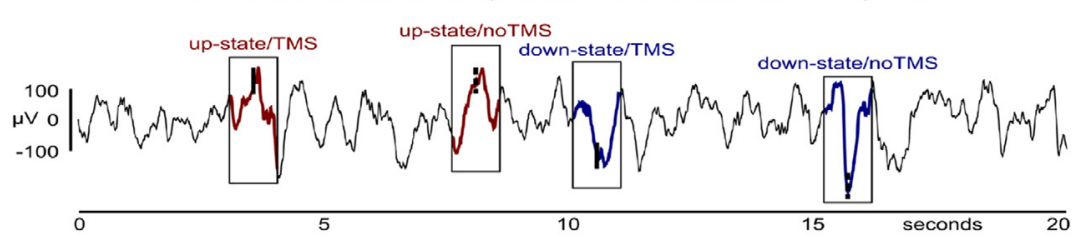

B. TMS outcome as a function of brain state

B1. Online triggering

MEP (peripheral response)

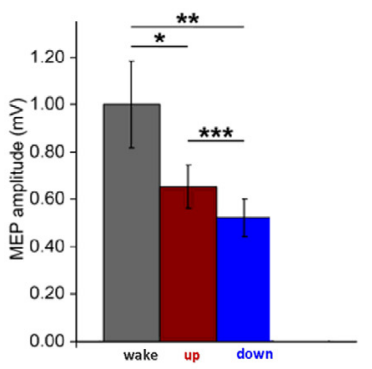

TEP (cortical response)

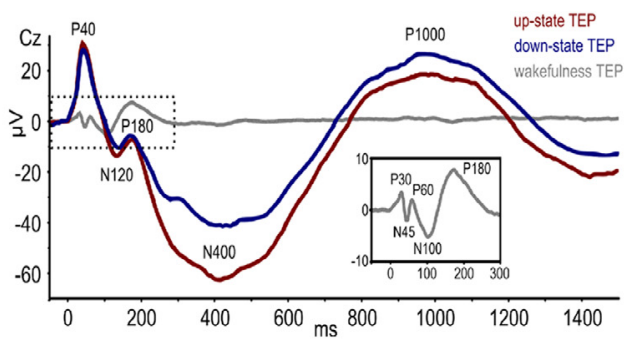

B2. Single-trial correlations (MEP) or binning (TEP) according to EEG amplitude MEP (peripheral response) TEP (cortical response)
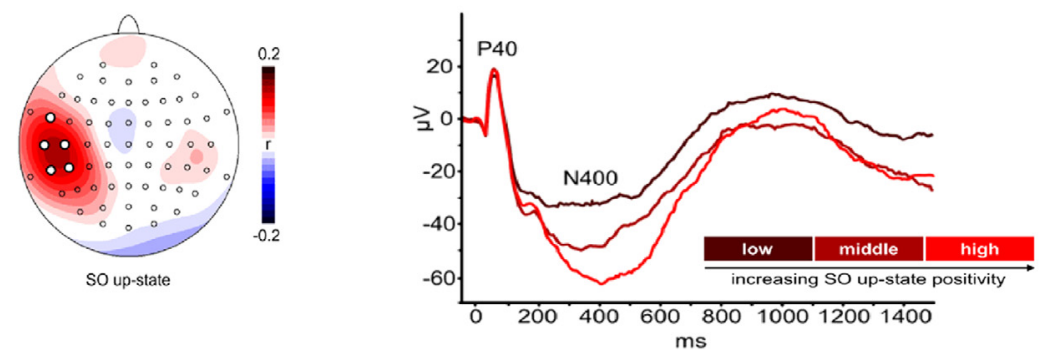

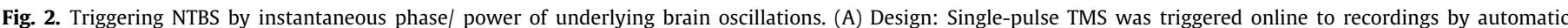

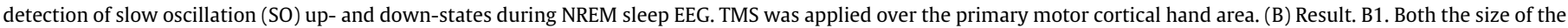

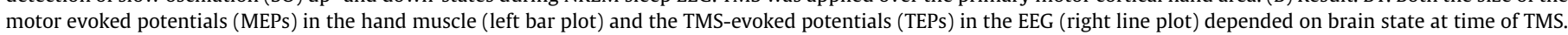

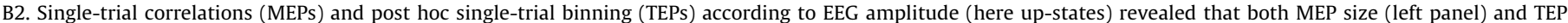
amplitude (right panel) scale with the EEG amplitude (i.e., actual voltage) at the time of TMS. Reproduced from Bergmann et al. (2012a) with permission.

TACS (for TMS examples see, Klimesch et al., 2003; Sauseng et al., 2009b; Romei et al., 2010, 2015; Thut et al., 2011; Chanes et al., 2013, 2015; Hanslmayr et al., 2014; Ruzzoli and Soto-Faraco, 2014; Jaegle and Ro, 2014; Quentin et al., 2015a,b; for TACS examples see, Pogosyan et al., 2009; Feurra et al., 2011; Joundi et al., 2012; Neuling et al., 2012; Santarnecchi et al., 2013; Helfrich et al., 2014b; Cecere et al., 2015; Witkowski et al., 2016; Chander et al., 2016; Ruhnau et al., 2016; Guerra et al., 2016). However, most of the evidence for the existence of entrainment effects comes from behavioral studies. In contrast, only very few TMS or TACS studies so far have managed to simultaneously record EEG/ MEG (due to contamination of the recorded neurophysiological signals by stimulation-induced artifacts). Even fewer have combined the two, i.e. recorded EEG/MEG and documented the associated behavioral effects. However, online registration of the EEG/ MEG signal is required to verify NTBS interaction with brain oscillations (here entrainment) as the basis of the behavioral change (see Section 4).
Some of the behavioral studies indirectly supporting entrainment by frequency-tuned TACS show performance measures in specific tasks (e.g. sensory detection) to co-cycle with the applied rhythmic electromagnetic force (e.g. over sensory areas) (Neuling et al., 2012). Similarly, TMS-probed excitability in intracortical circuits as inferred from paired-pulse designs (Hallett, 2007) shows modulation by TACS in a frequency- and phase-specific manner, both in terms of intracortical facilitation (ICF) and short-interval intracortical inhibition (SICI) (Guerra et al., 2016). Some level of entrainment seems to have modulated task performance and cortical excitability in-line with TACS phase, as suggested by these behavioral data. Other behavioral studies revealed that when rTMS or TACS is frequency-tuned to known, task-related oscillations, associated behavioral performance measures are biased in expected directions, i.e. in line with known correlative brainbehavior relationships (Klimesch et al., 2003; Sauseng et al., 2009b; Romei et al., 2010; Hanslmayr et al., 2014; Chanes et al., 2013, 2015; Quentin et al., 2015a; Pogosyan et al., 2009; Joundi 
et al., 2012), suggesting that rTMS or TACS has interacted selectively with the target oscillations and associated function by synchronization.

Using concurrent EEG, others have managed to demonstrate entrainment of brain oscillations during frequency-tuned rTMS. For instance, entrainment of parietal alpha oscillations has been demonstrated during short bursts of alpha-rTMS ( 5 pulses at individual alpha frequency) targeting the right intraparietal sulcus (IPS) (Thut et al., 2011), and entrainment of prefrontal beta oscillations was observed for a few cycles briefly after the end of stimulation when targeting the left inferior frontal gyrus (IFG) (Hanslmayr et al., 2014). Entrainment during short-burst rTMS is frequency-specific, as reflected in stronger entrainment for stimulation at individual frequencies than flanker frequencies (so far tested for beta-rTMS over motor cortex, see Romei et al., 2015). But are TMS-evoked oscillations actually generated by the same neuronal circuits as the targeted spontaneous oscillations? Support for this assumption comes from recent work demonstrating that alpha oscillations evoked by single-pulse TMS of the visual cortex were modulated by top-down attention in the same direction as spontaneous alpha oscillations, namely increasing in amplitude when visual attention was low and decreasing when it was high, which is opposite to the direction e.g. visual evoked potentials would be modulated (Herring et al., 2015). These studies, therefore more firmly establish entrainment of natural brain oscillations as a possible mechanism underlying the above described behavioral effects (see also Section 5).

For the case of TACS, there is clear electrophysiological evidence from animal work that entrainment is possible (Fröhlich and McCormick, 2010; Ozen et al., 2010), but also evidence for transcranial entrainment in humans is accumulating: Concurrent TACS-EEG data suggests that TACS is able to entrain occipital alpha oscillations, although sophisticated TACS-artifact removal procedures are required to extract the brain signals (Helfrich et al.,

\section{A. Entrainment of oscillations with alpha-rTMS/TACS over posterior alpha generators}

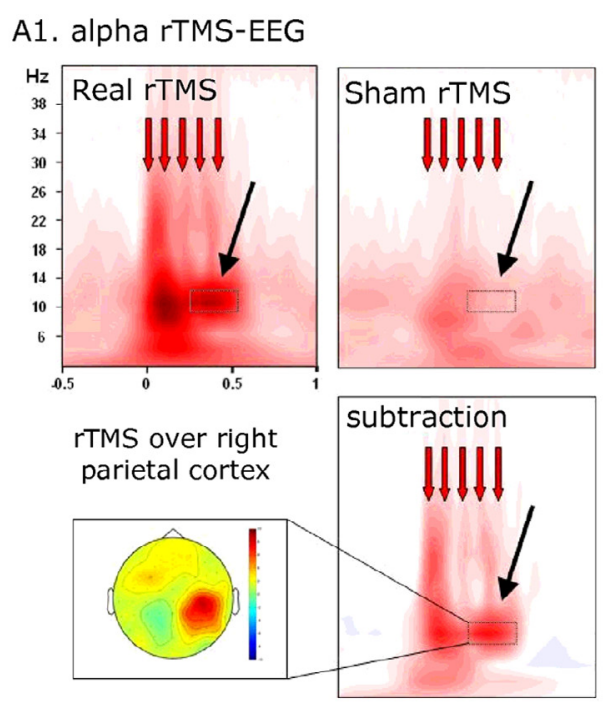

B. Functional consequences on behavior
A2. alpha TACS-EEG

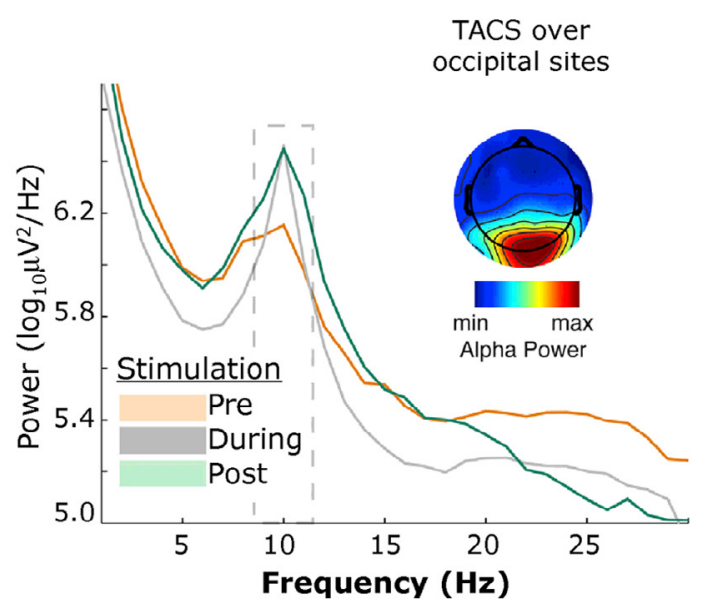

B1. alpha-rTMS \& perception

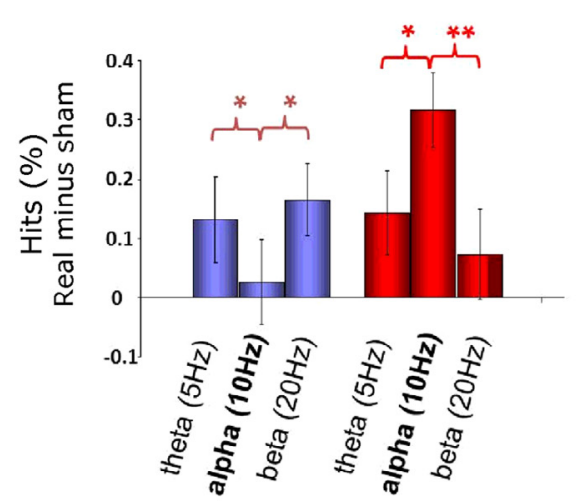

vis. target contralateral to TMS

vis. target ipsilateral to TMS
B2. alpha-TACS \& perception

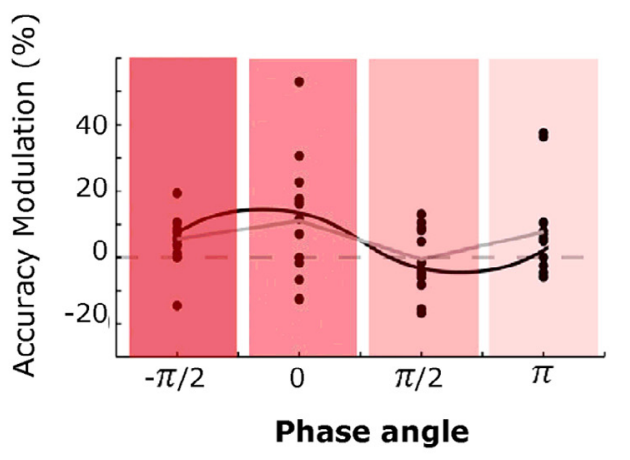

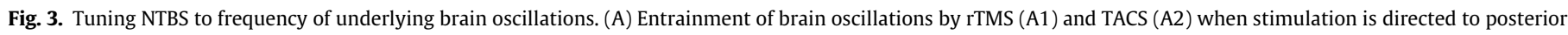

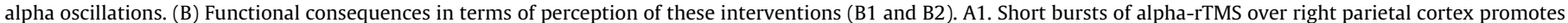

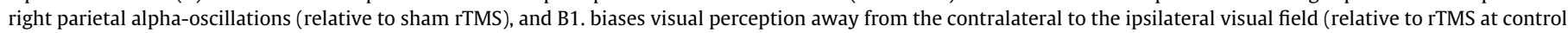

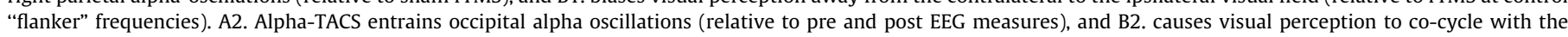
entrained alpha rhythm. Reproduced from Thut et al. (2011), Romei et al. (2010) and Helfrich et al. (2014b) with permission. 
2014b; but see Noury et al. (2016)). Pioneering works on concurrent TCS-MEG demonstrated the feasibility of recording MEG online to TCS, which (in contrast to TCS-EEG) allows to record oscillations directly from the target brain region underneath the stimulation electrode (Soekadar et al., 2013a; Neuling et al., 2015; Ruhnau et al., 2016). After it was shown that monosinusoidal TACS-EEG/MEG recording is accompanied by various stimulationand heartbeat-related artifacts (Noury et al., 2016) that are difficult to remove by any established methods (Marshall et al., 2016; Noury et al., 2016), a stimulation protocol was recently introduced that avoids the previously described artifact problems by using an amplitude-modulated TACS signal (Witkowski et al., 2016). It was shown that this protocol could entrain prefrontal midline theta oscillations affecting working memory performance and taskdependent theta power-regulation (Chander et al., 2016).

Together with the above described frequency-specific effects on behavior, these EEG/MEG data are suggestive of the possibility to control oscillatory activity and associated performance measures by frequency-tuned interventions. Examples of studies on frequency-tuning rTMS/TACS to brain oscillations are provided in Fig. 3, including evidence for entrainment in EEG (Fig. 3A) and for meaningful behavioral changes resulting from these interventions (Fig. 3B).

In addition to these immediate effects of NTBS, after-effects have been reported with frequency-specific TACS as well as frequency-specific rTMS. For example, entrainment of spontaneous alpha oscillations via TACS (Herrmann et al., 2013) may result in subsequent increases in alpha power (Zaehle et al., 2010; Neuling et al., 2013; Vossen et al., 2015), a phenomenon, which may indirectly rely on STDP induction in the specific alpha-generating circuits by entrainment, but is not a direct sign of entrainment itself (Zaehle et al., 2010; Vossen et al., 2015, see also Veniero et al. (2015)).

\subsection{Combinations of frequency-tuned (3.2) and phase-triggered (3.1) interventions}

It is likely that the above frequency- and phase-tuned interventions may be potentiated when combined (Fig. 1C, right panel). One potentially effective variant of this combination is to lock frequency-tuning to specific oscillatory phase angles or power values of ongoing oscillations (using real-time NTBS-EEG/MEG approaches). Indeed, the strength of entrainment of parietal alpha oscillations during frequency-tuned rTMS depends on the alphaphase at which the rTMS-train catches the ongoing alphaoscillation, as revealed by post hoc trial sorting (Thut et al., 2011). Similarly, strength of alpha-entrainment during alphaTACS seems to depend on the ongoing alpha-power (eyes-open vs. closed) (Ruhnau et al., 2016). Although real-time power- or phase-dependent frequency-tuning will likely be advantageous, no study has implemented this approach so far.

A second variant of combining frequency- and phase-tuning is to entrain brain oscillations with frequency-tuned interventions (e.g. TACS), together with the presentation of discrete events (e.g. single TMS pulses, gamma bursts, etc.) at specific NTBS phase angles. The feasibility of this approach has recently been shown for TACS-TMS over motor cortex (Raco et al., 2016). Others have combined two TACS waveforms (Alekseichuk et al., 2016) to emulate the circuit motif of cross-frequency phase-power couplings, reported in many EEG/MEG-studies (see Section 2 above). To this end, Alekseichuk et al. (2016) applied TACS over frontal areas in a cross-frequency regime, while participants were performing a working memory task. Fast gamma-TACS stimulation signals were superimposed on a slower, background theta-TACS oscillation (Fig. 4A), which led to marked changes in working memory performance (Fig. 4B) and brain connectivity (Fig. 4C), depending on the phase gamma-TACS was locked to. Importantly, this was not observed with the gamma-TACS bursts just repeated at theta rate (see Fig. 4).

Other combinations are conceivable, e.g., testing whether the pulses of plasticity inducing TMS protocols would be more effective if tuned to specific phases of simultaneously applied TACS, in particular when the latter is frequency-tuned to physiologically meaningful oscillations (see Goldsworthy et al., 2016). This is analogous to the idea that the efficacy of these protocols may be enhanced if TMS pulses are phase-locked in real-time to EEGsignals (see Section 3.1 above).

A third, related approach relies on the simultaneous, frequencytuned intervention with two nodes of a network (e.g. by doublesite TACS), combined with phase-alignment of these two interventions. The approach is about phase-coupling or phase-decoupling of the two, spatially separated TACS stimulation signals to potentially promote or suppress communication between the two stimulated nodes of the network (in alignment with the principle of communication-through-coherence (Fries, 2005), see also Section 2). Examples can be found in Polania et al. (2012) and Helfrich et al. (2014a). These studies tuned TACS frequencies to the natural rhythm of the network under study, e.g. to theta of a fronto-parietal network (Polania et al., 2012) or gamma of a bihemispheric occipital network (Helfrich et al., 2014a). The results suggest that the phase-lag between the two TACS waveforms (inphase versus out-of-phase) affects the associated functions (i.e., working memory or perception of horizontal motion $)^{1}$ in line with the notion of interfering with functional connectivity by network coupling/decoupling via double-site TACS.

\section{Methodological considerations: proper documentation of effects}

The EEG/MEG-informed NTBS approach outlined above relies on principled ideas about the relevance of intrinsic brain oscillations in shaping brain function, and how to interact with them. More specifically, both spontaneously fluctuating and NTBS-regulated brain oscillations are thought to represent modulators of the behavioral outcomes of NTBS, when appropriate timing and frequency of NTBS relative to the oscillations are used. Accordingly, it is important to document not only the behavioral outcome but also the hypothesized electrophysiological underpinnings of this approach, which requires the recording of EEG or MEG simultaneously to the intervention. For instance, for phase-tuned intervention, it is important to verify proper phase-targeting in EEG/MEG, while for frequency-tuned intervention, entrainment should ideally be demonstrated, alongside the behavioral effects. However, there are important challenges in the documentation of the electrophysiological underpinnings of these effects, depending on the chosen protocol (e.g. TMS vs. TACS) and effects of interest (immediate vs. after-effects), mainly due to NTBS-induced artifacts in the EEG/MEG recordings as well as EEG/MEG contaminations due to NTBS-associated peripheral sensations. Below we outline these

\footnotetext{
${ }^{1}$ It should be noted that studies using a single "return" electrode (e.g., vertex) for two "active" electrodes (e.g., frontal and parietal) to produce in-/out-of-phase conditions (e.g., Polania et al., 2012) not only vary the phase-lag between the two active sites, but also vary the direction of current flow in the brain tissue: The current flow is fronto-parietal (and vice versa) when both sites are out-of-phase (since in that case the two sites have opposite polarity), but fronto-vertex and parieto-vertex (and vice versa) when the two active sites are in-phase (i.e., same polarity) but out-ofphase with the vertex (i.e. different polarity). This problem is circumvented to a certain degree with local center-surround montages (e.g., Helfrich et al., 2014a), where it can at least be assumed that less current flows between the two local montages. For other, useful electrode options in this regard, see also Bortoletto et al. (2016).
} 

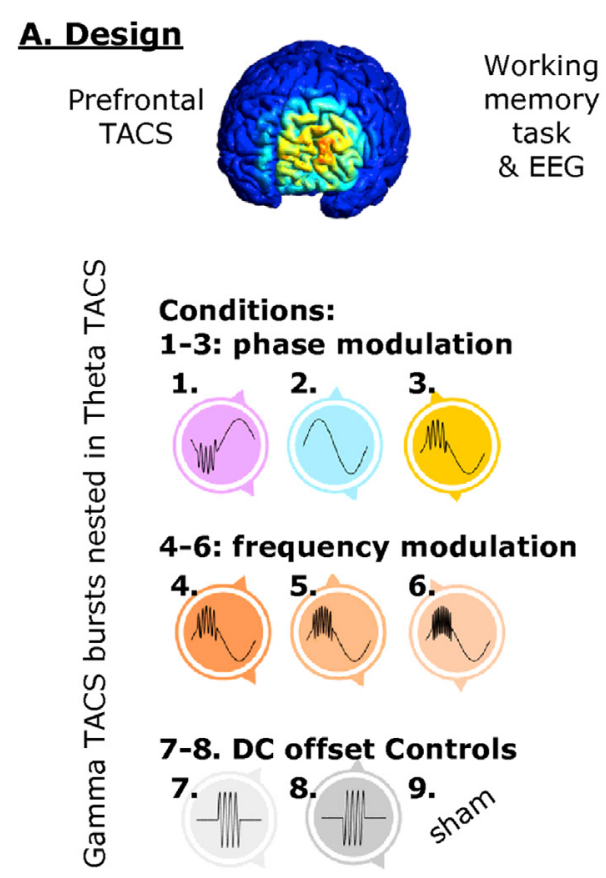

\section{EEG effects}

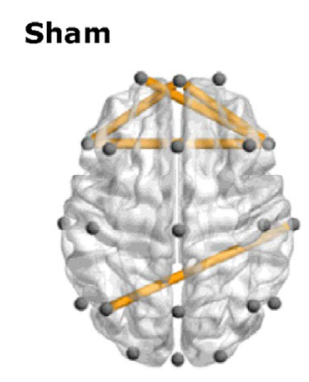

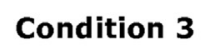

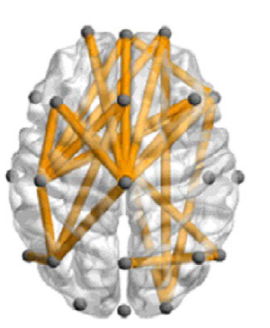

\section{B. Behavioural effects}

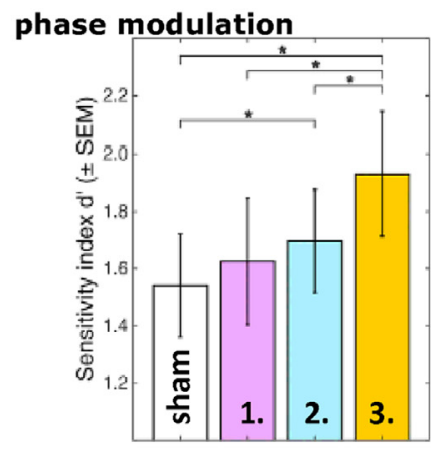

frequency modulation

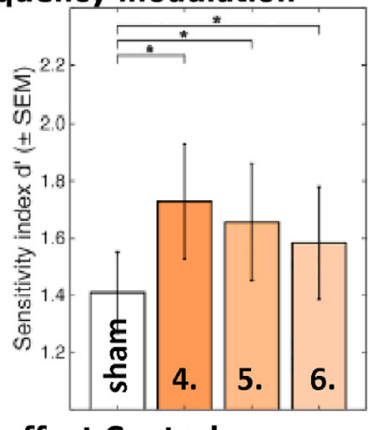

DC offset Controls

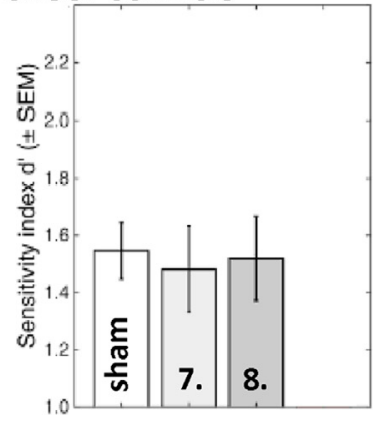

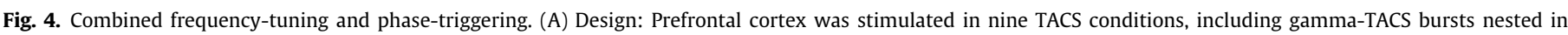

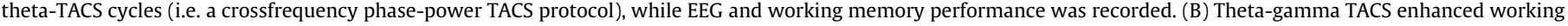

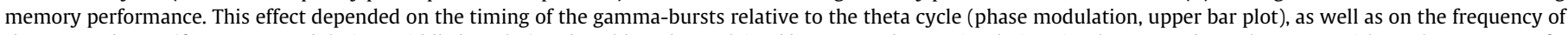

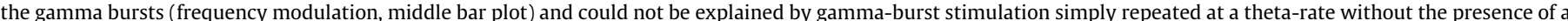

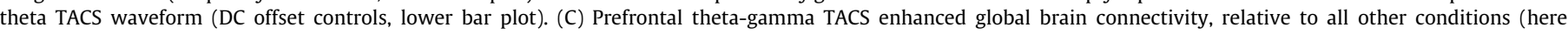
illustrated for sham). Reproduced from Alekseichuk et al. (2016) with permission.

challenges and the experimental designs that allow controlling for them, which are critical for evaluating the success of the approach.

\subsection{Documentation of behavioral effects}

The assessment of behavioral effects is important in the first place, as these effects are the primary outcome measures of most experimental and clinical interventions. The experimental designs should be chosen to allow testing the benefit of adding a temporal to the spatial dimension of targeting. This can be achieved by implementing appropriate control conditions mimicking conventional approaches, e.g., phase jittering stimulation or use of arbitrary (but non-harmonic) stimulation frequencies relative to the target oscillation.

\subsection{Documentation of electrophysiological underpinnings}

Documentation of online interactions of NTBS with brain oscillations as the origin of the behavioral effects is problematical because of the NTBS induced electrical artifacts in EEG/MEG online to stimulation. For TMS, these consist of brief but high amplitude deflection in the EEG which can be minimized by using appropriate hardware (Virtanen et al., 1999; Veniero et al., 2009) and further reduced by additional, post hoc artifact reduction procedures (Siebner et al., 2009; Ilmoniemi and Kicic, 2010; Vernet and Thut, 2014). For TACS, the electrical artifact is likewise of high amplitude (reaching $\mathrm{mV}$ levels relative to $\mu \mathrm{V}$ neuronal signals) but, in addition, is present continuously, which renders it more resistant to elimination (Noury et al., 2016). On top of these electrical artifacts, each technique is associated with a set of unwanted peripheral sensations. For TMS, these consist of auditory and tactile sensations (and associated cranial muscle potentials) (Nikouline et al., 1999; Mutanen et al., 2013; Rogasch et al., 2014). For TACS, the main physiological contaminations are visual sensations originating from stimulation of the retina (Schwiedrzik, 2009; Schutter and Hortensius, 2010; Schutter, 2016; Laakso and Hirata, 2013), which are frequency-dependent (Turi et al., 2013) and occur with many electrode montages due to the retina's high sensitivity to 
electrical currents. The same concern pertains to electrical stimulation of the cochlea, of the vestibular system in general, and of sensory afferents in the skin. Importantly, these TMS- and TACSinduced sensory responses and associated evoked potentials may themselves interact with brain oscillations and hence confound TMS/TACS outcome, even when below the subject's perceptual threshold. Therefore, to demonstrate interaction with ongoing oscillatory activity as the origin of the observed behavioral effects requires effective electrical artifact reduction and a number of appropriate active control conditions to rule out sensory confounds.

Electrical artifacts reduction algorithms have been proposed for TMS-EEG (Ilmoniemi and Kicic, 2010; Vernet and Thut, 2014, see also Rogasch et al., 2016: https://nigelrogasch.github.io/TESA and Herring et al., 2015: www.fieldtriptoolbox.org/tutorial/tms-eeg for removal pipelines implemented in EEGlab and FieldTrip). Likewise, such algorithms have been implemented for TACS-EEG (Helfrich et al., 2014b, for limitations see Helfrich et al., 2014a) and TACS-MEG applications (Soekadar et al., 2013a; Neuling et al., 2015). For TMS-EEG, a recent study could convincingly show that provided appropriate artifact reduction procedures are followed, TMS-evoked potentials are absent from EEG in patients with extensive cortical lesions when damaged tissue is stimulated, but intact when the functional portion of cortex was targeted (Gosseries et al., 2015), suggesting that electrical artifacts can effectively be eliminated by existing procedures. Likewise, recent TACS-EEG studies evaluating event-related potentials in response to sensory stimuli (Helfrich et al., 2014b) or physiological brain activity patterns (in terms of topography and reactivity to eyesopen and closed conditions) (Neuling et al., 2015) suggest effective artifact reduction despite simultaneous electrical stimulation. In general, however, artifact reduction algorithms often require computationally heavy processing steps (including independent component analysis (ICA), source estimates or data interpolation). This allows for retrieving the EEG/MEG signal offline to the recordings but is yet incompatible with real-time analyses and interventions in many cases. For real-time applications, alternative neurophysiological read-outs or stimulation procedures circumventing the artifacts have therefore been used (see Section 6.1 below). Furthermore, while several procedures for artifact reduction exist, future research is needed for further evaluation and improvements.

In addition, control conditions should be designed to equate the potential sensory confounds of TMS and TACS, which may interact with brain oscillations by themselves (e.g., cause entrainment). Ideally, active controls should be used that are as similar as possible to the main condition in terms of the sensory component but less effective in regards to transcranial cortical stimulation. For TMS, this could consist of rotating coil orientations to a less effective direction of current flow (Thut et al., 2011) or the use of extracranial control sites like the shoulder blade (Herring et al., 2015) that provide comparable multisensory (auditory and tactile) inputs. For TACS, active control montages are likewise desirable, since the retina, inner ear, and peripheral sensory and vestibulocochlear nerves have low stimulation thresholds, as a result of which even subliminal (unperceivable) stimulation may affect brain oscillations and confound "transcranial" cortical effects (Utz et al., 2010; Schutter, 2016). Control montages can include extracephalic return electrodes, or when possible, the use of other stimulation frequencies that are behaviorally not relevant.

For the documentation of entrainment effects, control conditions with different temporal patterns should also be considered, including stimulation at different frequencies, e.g., at both higher and lower neighboring frequencies (Romei et al., 2010, 2015; Chanes et al., 2013), at arrhythmic (trial to trial randomized) pulse timings or rhythmic irregular intervals (fixed pulse timing at unequal intervals) (Thut et al., 2011; Chanes et al., 2013, 2015; Quentin et al., 2015b), or using another montage stimulating task-irrelevant areas but at the target frequencies. If TACS has a DC-offset (so called oscillatory TDCS, oTDCS), there needs to be a control for mere effects of DC, as oTDCS should have the same effects as TDCS as long as total charge is matched (Bergmann et al., 2009; Groppa et al., 2010).

After-effects of NTBS on physiological parameters are easier to demonstrate due to the lack of artifacts in the critical (i.e. preand post-stimulation) time periods. Nonetheless, the same control conditions as discussed above need to be implemented, because confounds during stimulation may also affect after-effects.

\section{Open question: mechanisms of interventions}

Fundamentally, the effect of rTMS and TACS on neuronal dynamics of oscillatory brain activity remains mostly unknown. This is because online monitoring of brain activity during stimulation is technically challenging and the extrapolations from the low number of studies using animal model preparations and computer simulations are not straightforward. Despite its limitation, the conceptual model which has gained the most traction for the response of brain networks to periodic (frequency-tuned) NTBS focuses on entrainment (Thut et al., 2011). Most generally spoken, entrainment refers to the behavior of an oscillating system to a periodic perturbation, where the system "locks" to the stimulation such that its frequency shifts to the frequency of the applied stimulation (or a harmonic/subharmonic). Certain conditions must be met for a periodic perturbation to accomplish successful entrainment. The stronger the intensity of stimulation, the broader is the range of frequencies (centered at the endogenous frequency in absence of stimulation) at which the network can be entrained. This principle is referred to as the Arnold tongues and has been well described for (quasi-)linear systems subjected to an external periodic force. It is important to note that while such Arnold tongue behavior has been shown in computer simulations (Ali et al., 2013; Herrmann et al., 2016), experimental evidence from animals or humans is limited due to the required number of stimulation intensities and frequencies that need to be evaluated.

For example for TMS, the relationship between stimulation intensity and potential phase resetting has not been sufficiently characterized yet. TMS at lower intensities or in the presence of strong endogenous oscillations may exert a phase-dependent effect while leaving the ongoing oscillation relatively unaffected. Conversely, at sufficiently high intensities or for weak endogenous oscillations TMS may phase-reset the circuits generating the endogenous oscillation and result in a TMS-locked oscillation. Importantly, in the latter case phase-reset would not necessarily be expected for the entire ongoing oscillation (observed from the summed potential/field EEG or MEG recordings), but rather for a circumscribed local population of stimulated neurons. While being largely unknown, the relationship between stimulation intensity and phase-resetting/entrainment is of relevance for both the EEG-triggered informed open-loop and fully closed-loop approaches (see next section).

In addition, it is important to note that the underlying assumption of brain network oscillations to reflect measures generated by quasi-linear signals does not necessarily hold. For example, stimulation with periodic pulse trains at $10 \mathrm{~Hz}$ in epilepsy patients implanted with subdural electrode arrays for clinical monitoring revealed that entrainment may occur in the case of a relative weak endogenous oscillation, which is more susceptible to perturbations, whereas in states of pronounced endogenous oscillations the effect of the stimulation is not a shift to the stimulation frequency (as would be expected with entrainment), but rather an 
increase in power at the endogenous frequency (Alagapan et al., 2016). This phenomenon can be easily explained by simple nonlinear threshold models, but not by the more commonly used Arnold tongue framework (Alagapan et al., 2016). In conceptual agreement, in a reduced slice preparation that combined optogenetic activation of the network with electric field application, only for weak optogenetic activation did the electric field enhance the activity at the stimulation frequency (Schmidt et al., 2014).

As to the mechanisms underlying changes in brain oscillation by amplitude-modulated TACS (as opposed to mono-sinusoidal TACS) in which the amplitude of a high-frequency carrier signal is modulated at a frequency of interest (Witkowski et al., 2016), it was discussed that the entrainment effect of such protocols might be related to non-linear properties of cell membranes (Goldman, 1943) resulting in rectification of the TACS signal. In this context, configurational changes of membrane proteins that lead to modifications of ionic binding sites and membrane permeability may play an important role.

\section{Future perspective: optimization of the approach}

While there is emerging evidence for the interest of informing NTBS by EEG/MEG in terms of timing and frequency, many of the reported effects still await replication and need to be evaluated as to whether their effect sizes lend themselves to clinical applications and/or can be further amplified. In this endeavor, the choice of optimal stimulation parameters will be important. While timing and frequency is informed by EEG/MEG, many other stimulation parameters are normally chosen arbitrarily (due to a lack of knowledge on how to guide them). Since the parameter space to choose from is almost infinite, including (i) intensity of stimulation for TMS and TACS, (ii) pulse form and coil orientation for TMS, (iii) electrode montage and stimulation waveform (sinusoidal, saw-tooth, rhythmic squared, i.e. pulsed, amplitude-modulation) for TACS, (iv) number of pulses or duration of stimulation, ( $v$ ) stimulation of resting state vs. stimulation during a task etc., an exploration of the whole parameter space for finding the best parameter configuration is likely unfeasible. The development of optimization strategies will therefore be an important line of future research. For interactions with brain oscillations, two such strategies have been proposed, namely real-time closed-loop interventions and guidance by biological plausible models, as outlined below.

\subsection{Data-driven optimization: real-time open-loop versus closed-loop} approaches

Recent technical advances enable the use of informed openloop and even fully closed-loop approaches, which evaluate the EEG (or in principle also MEG) signal in real-time to control the concurrent NTBS application accordingly (Bergmann et al., 2016; Zrenner et al., 2016): informed open-loop exploits amplitude and phase information of a specific ongoing oscillation in order to trigger stimulation in a temporally specific manner, however, without aiming to change the underlying oscillation. In contrast, a fully closed-loop approach aims to alter the targeted neuronal activity (Karabanov et al., 2016), e.g., by increasing or decreasing the amplitude of oscillatory brain activity or phase-locking it to the stimulation. See Fig. 5 for a schematic representation. Provided that brain oscillations are effective targets for NTBS, these approaches are expected to help optimizing interventions.

The first study using EEG-triggered TMS in an informed openloop manner quantified motor cortical excitability and TEP changes during different phase-angles of the slow $<1 \mathrm{~Hz}$ NREM sleep oscillation (Bergmann et al., 2012a). Nowadays, oscillatory phase angles can be assessed with even higher temporal precision and shorter time delays, thus also allowing to target faster oscillations in
A. Open-loop stimulation

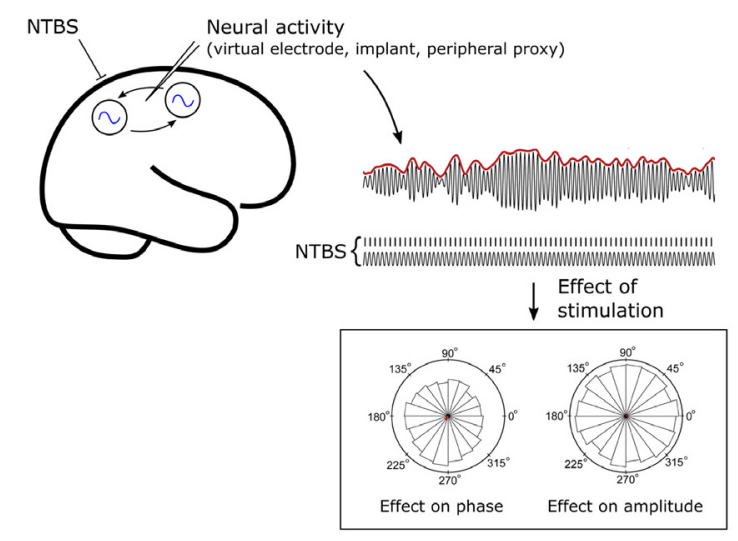

B. Closed-loop stimulation

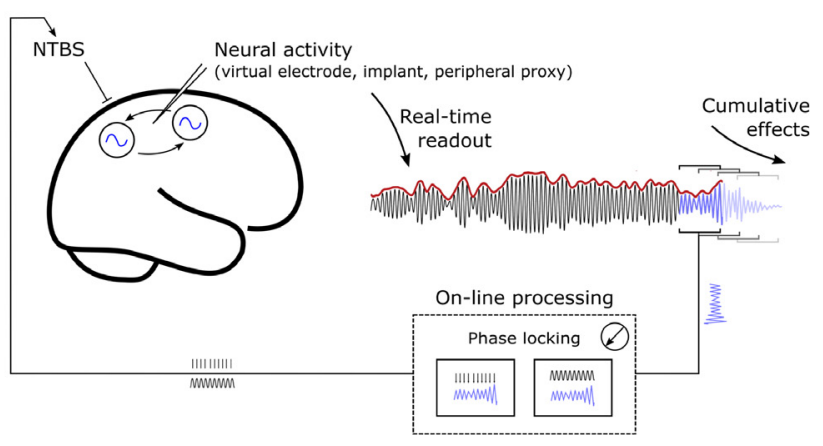

Fig. 5. Control of NTBS. (A) Open-loop stimulation. Neural activity is extracted by signal processing techniques (e.g. beamforming in EEG/MEG), directly from neural implants, or inferred from a peripheral proxy such as muscle activity. The relationship between neural activity and stimulation waveform is then calculated (offline) to determine the influence of stimulation on, for example, the phase and amplitude of the endogenous neural activity. (B) Closed-loop stimulation. Neural activity is readout in real-time and processed to determine the appropriate form of stimulation on a moment-by-moment basis. On-line processing is technique dependent, such as targeting specific phase points via TMS, or providing continuous feedback via phase locking in the case of TACS. In either case, closed-loop stimulation requires knowledge of target parameters (such as the optimal choice of phase) that may come from an a priori hypothesis, or be determined empirically by open-loop stimulation. Fully-closed loop approaches aim to enhance (or suppress) neural synchrony within- or between- target populations.

real-time (Zrenner et al., 2010, 2015, 2016; Triesch et al., 2015). It needs to be noted however that depending on sampling rates, communication protocols or data pre-processing steps (e.g. head localization in MEG), timing delays and jitter may occur, which can impede precise timing of stimulation.

To date, no closed-loop NTBS has been demonstrated using EEG that relies on simultaneous read-out and targeting of activity in neural population (Karabanov et al., 2016). For a recent study that relies entirely on activity in neural population but using read-out in stimulation-free intervals only see Lustenberger et al. (2016). This limitation is predominantly due to the considerable stimulation artifact in EEG recordings caused by either TMS or TACS, which interferes with real-time assessment of the oscillatory target parameters, once stimulation has started (for a detailed discussion see Bergmann et al., 2016). Instead, closed-loop strategies with EEG recordings have thus far employed either indirect read-outs of neural activity (e.g. concentrating on the behavioral consequences of the stimulation), or non-electrical forms of stimulation that circumvent stimulation artifact entirely. For instance, Ngo et al., 2013, 2015) delivered auditory tones for interventions during sleep. The tones were phase-triggered to slow sleep oscillations 
with the aim to enhance these oscillations, facilitating overnight memory consolidation. In another example using motor cortex TACS to reduce tremor in Parkinson's disease, actigraphy from the tremulous limb was adopted as a proxy for central neural activity (Brittain et al., 2013). The approach involved an initial openloop followed by a closed-loop intervention that afforded several key advantages: First, knowledge of phase information for both TACS waveform and target oscillations (made possible by the proxy measure of central activity) permitted a direct measure of entrainment (Mehta et al., 2014, 2015; Brittain et al., 2015). Second, the phase-precession associated with open-loop stimulation permitted the construction of a stimulus response profile, in order to identify the most effective stimulation parameters for tremor suppression. Third, the phase of the target oscillation could then be used to directly inform real-time closed-loop (phase-locked) stimulus delivery. Indeed, stimulus response profiles revealed that (for tremor at least) it was the phase-difference between stimulation and tremor oscillations - rather than the phase of stimulation itself - which was the crucial factor in selectively suppressing or exacerbating the peripheral tremor. Of course, this approach relied on the hypothesis of a strong (causal) relationship between central oscillatory rhythms and peripheral outflow, surmised from prior functional connectivity studies (Timmermann et al., 2003). Yet despite this caveat, the closed-loop TACS saw a marked increase in effect-size relative to open-loop stimulation (Brittain et al., 2013). Finally, as an alternative real-time intervention approach, temporal interleaving of stimulation and recording epochs may also be considered. This approach has been shown to successfully control alpha oscillations using TACS in an early report (Boyle and Fröhlich, 2013) and was recently used to detect oscillatory transients in real-time (namely sleep spindle activity during NREM sleep) to then engage the target oscillation by individualized TACS limited to brief time epochs, enhancing not only sleep spindle activity but also overnight memory consolidation (Lustenberger et al., 2016). In addition, feasibility of NTBS during online MEG (instead of EEG) was successfully demonstrated and allowed a chronic stroke patient to modulate ipsilesional sensori-motor rhythms (SMR) while tDCS was applied to the ipsilesional sensori-motor cortex (Soekadar et al., 2013b). This study suggests that also MEG source activity-informed tACS will be feasible (requiring a real-time MEG system, though).

As a cautionary note, it needs to be mentioned that it is unknown how the sustained cumulative effects of closed-loop stimulation delivered under steady-state conditions (such as during bouts of tremor) would translate to behavioral paradigms, where fluctuating neural dynamics are constantly being reset and updated in a context and state-dependent manner. In addition, in several studies entrainment has been reported to be weak (Mehta et al., 2014, 2015; Brittain et al., 2015). Since open-loop protocols rely on assumptions about underlying mechanisms (e.g. steady entrainment of ongoing oscillations), there is a danger that such protocols will be undermined by unobserved temporal relationships and dynamic changes in the course of stimulation (e.g. due to homeostatic plasticity), even when TACS is delivered at the (subject-specific) natural resonance frequency of the targeted neuronal circuit. The advent of informed open-loop and closedloop approaches linked to real-time electrophysiological readouts (such as EEG and/or MEG) therefore appears to offer a crucial step forward in optimizing stimulation protocols.

\subsection{Model-driven optimization: prediction of effects with biologically} plausible models and simulations

Another promising, emerging means for guiding intervention is computational modeling of stimulation effects, also referred to as computational neurostimulation (Bestmann, 2015) (for TMS see
Rusu et al., 2014; Hartwigsen et al., 2015; Triesch et al., 2015; for TACS see Fröhlich, 2015). These models implement a physiological component to work towards a mechanistic understanding of the NTBS-brain interaction, and are complementary to e.g. anatomically realistic finite element models that estimate actual intracranial field distributions (Opitz et al., 2015), which may be used to correct for substantial inter-individual variability in this measure (Opitz et al., 2016). In the modeling approach, mathematical models of the targeted neuronal networks can be subjected to stimulation in computer simulations allowing to take into account several parameters (e.g. spatial and temporal) of NTBS. In contrast to human or even animal studies, computational modeling therefore allows for the rapid evaluation of a large number of stimulation parameter combinations in terms of their effects on network dynamics. Such comprehensive parameterization has enabled the identification of entrainment of brain oscillations (due to the presence of Arnold tongue behavior, see Section 5 above), as a fundamental mechanism of target engagement by TACS (Ali et al., 2013; Herrmann et al., 2016). This is particularly important for TACS, where the small magnitude of the electric field delivered to the brain requires a synergistic interaction with endogenous network dynamics for the stimulation to have an effect (Fröhlich, 2015). In addition, mathematical models of sufficient biological plausibility enable the identification of cellular mechanisms that contribute to the effect of TACS. While there is certainly a broad range of computational models that can help to elucidate the interaction between the network dynamics targeted by stimulation and the applied stimulation, models that accurately model the non-linear dynamics for membrane voltage values around the action potential "threshold" may be of particular importance since this non-linearity likely enables the small changes in membrane voltage induced by NTBS to have an effect on the spiking of individual neurons and thus the network (Bonaiuto and Bestmann, 2015).

\section{Future perspective: the promise of multimodal neuroimaging (TMS-EEG-fMRI)}

As described above, electrophysiological methods such as EEG/ MEG are capable of noninvasively measuring the temporal aspects of ongoing neuronal activity and their fluctuations, thereby capturing various parameters of neural oscillations, which may then be used to guide NTBS for optimizing when and how to stimulate. Complementing this focus on the temporal domain, functional magnetic resonance imaging (fMRI) - the established noninvasive method for measuring the spatial aspects of function-related neural activity - allows examining activity changes in localized brain regions and networks across the whole brain. Much has been written about the various neuroimaging tools and their complementary benefits, and it is widely accepted that combining them provides, at least conceptually, a rich and relatively complete view on brain function at the macroscale.

'Multimodal imaging' generally involves a two-way combination of tools, such as fMRI-EEG, fMRI-TMS, fMRI-TACS, EEG-TMS, or EEG/MEG-TACS, which can encompass either offline or online (i.e. simultaneous) combinations (Siebner et al., 2009; Reithler et al., 2011; Ziemann, 2011; Bergmann et al., 2016). Such simultaneous combinations are challenging, both technically and in terms of analysis, but have added value over and above offline combinations (Siebner et al., 2009). The reason for this is straightforward: in simultaneous setups, the same participant can be measured in the same environment, position and mindset with the same fluctuations of attention, comfort, and equivalent influence of other extraneous variables. This increases validity and eliminates certain sources of noise. More importantly, simultaneous combinations 
allow us to uniquely address particular questions, and to approach data analysis in specific ways, in order to deliver more fundamental insights on the interaction of NTBS with brain activity and related behavior.

Here, we focus on the value of adding fMRI to EEG-TMS or EEGTACS research (i.e. the three-way combination approach). In a feasibility study, Peters et al. (2013) combined commercially available hardware for simultaneous TMS-fMRI and simultaneous EEG-fMRI to evaluate safety/comfort and signal quality using a variety of tests on phantom and human volunteers. The three-way simultaneous combination of fMRI, EEG and TMS was shown to be safe, tolerable, and to provide good-quality signals. This grants new opportunities for future research to learn about how oscillatory activity in different frequency bands relates to activity and interactions in large-scale functional brain networks, and how this may be modulated by TMS/TCS. In a first approach to combine EEG, TACS and fMRI, Vosskuhl et al. (2016) showed that tACS at the individual alpha frequency - as determined by EEG - down-regulates the visually event-related BOLD response but not the ongoing BOLD activity.

Simultaneous fMRI-EEG already allows meaningful multimodal integration, and to correlate fluctuations in oscillatory parameters (e.g. increases/decreases of power in a particular frequency-band) to fluctuations in network BOLD signals (Debener et al., 2006; Scheeringa et al., 2009, 2011; Bergmann et al., 2012b). But because the temporal scales of EEG and fMRI are so far apart (operating in the range of milliseconds versus seconds, respectively), this approach has limitations. The simultaneous three-way combination of TMS-EEG-fMRI may offer a unique way to look deeper into the relation between oscillatory parameters such as phase, power, and coherence, and network activity as measured by fMRI. This setup allows the integration of the spatial and temporal domain, using one imaging method for indexing brain state (e.g. EEG to determine ongoing oscillation parameters such as power and phase), while the complementary imaging method can serve as the actual read-out measurement (e.g. TMS-induced BOLD fMRI network effects). By this means, the modulation of TMS-probed cortico-subcortical network effects (revealed by TMS-fMRI) can be assessed as a function of ongoing cortical oscillation parameters (as indexed by concurrent EEG). In other words, TMS pulses are used as system probes, inducing a network response measured by fMRI, depending on the EEG-measured oscillatory brain state at the time of the pulse. Besides providing fundamental insight on network functions (in particular if combined with behavioral assays), the three way approach should help the understanding of NTBS-effects (and their state-dependency) in important ways. More specifically, one can ask the question to what extent TMSelicited BOLD responses throughout motor, perceptual, or cognitive networks are scaling with the momentary power, phase, or coherence of oscillations in functionally relevant frequency bands. Additionally, one can clarify how such EEG-fMRI network responses depend on functional state, such as rest versus task, different task conditions, or parametrically varied task loads, in order to provide fundamental insights into the relationship between oscillations, behaviorally relevant brain networks and NTBS effectivity.

\section{Conclusion}

We reviewed emerging ideas on how to work towards enhancing the specificity and effectivity of established NTBS protocols, based on the combination of NTBS mainly with EEG/MEG (and also fMRI). These ideas emphasize brain oscillations as key players in a number of fundamental circuit motifs that influence brain func- tions (Singer, 2009) and, as a consequence, constitute interesting targets for interventions. We identified three approaches that have been successfully used to interact with oscillatory brain activity: (1) triggering NTBS events to instantaneous phase- or powervalues of ongoing EEG/MEG that reflect states of heightened excitability, which is promising for enhancing NTBS effectivity; (2) tuning NTBS to the known frequencies of specific taskrelevant brain oscillations, in order to entrain these oscillations and promote the functions of the associated network; and (3) phase-triggering NTBS events to NTBS-entrained oscillatory brain activity, in order to potentiate approaches $1 \& 2$ by their combination. Initial results are promising, but further research is needed to document in more detail the electrophysiological underpinning of NTBS-induced network changes when guided by EEG/MEG for working towards a mechanistic account. The approach also affords the opportunity for implementing in future research both dataand model-driven optimization strategies (via real-time interventions and computational neurostimulation), which will be crucial for developing NTBS into an effective tool for experimental and clinical interventions into brain network activity and its (dys)functions. In brief, the outlined approach and ideas offer a framework for a hypothesis-driven, principled way of tailoring brain stimulation to interact with brain activity for shaping the brain-behavior relationship, constituting a promising new departure from conventional NTBS studies.

\section{Acknowledgments}

GT is supported by the Wellcome Trust [grant number 098434]. TOB is supported by the German Research Foundation via TR-SFB 654 ("Plasticity and Sleep"), and by the Hertie Foundation via the Hertie Institute for Clinical Brain Research. FF is supported by the National Institute of Mental Health of the National Institutes of Health under Award Number R01MH101547. The content is solely the responsibility of the authors and does not necessarily represent the official views of the National Institutes of Health. AV-C is supported by the IHU-ICM-Translationnal initiative \& Agence National de la Recherche Scientifique (ANR Génerique 2015 "Oscilloscopus"). ATS is supported by a grant from the Netherlands Organisation for Scientific Research (NWO VICI, grant number: 453-15-008). JSB is supported by the Medical Research Council (MR/N003446/1). HRS is supported by the Novo Nordisk Foundation (Interdisciplinary Synergy Programme Grant "BASICS" NNF14OC0011413). CSH and UZ are supported by the German Research Foundation (HE 3353/8-2 and ZI 542/7-1, respectively).

Conflict of interest: GT has received honoraria as editor from Wiley Publishers. FF is the lead inventor of IP filed by UNC. The clinical studies performed in the Frohlich Lab have received a designation as conflict of interest with administrative considerations. FF is the founder, CSO, and majority owner of Pulvinar Neuro LLC. HRS has served on a scientific advisory board for Lundbeck A/S, Valby Denmark, and has received honoraria as speaker from Biogen Idec, Denmark A/S, Genzyme, Denmark and MerckSerono, Denmark, has received honoraria as editor from Elsevier Publishers, Amsterdam, The Netherlands and Springer Publishing, Stuttgart, Germany, has received travel support from MagVenture, Denmark, and has received a research grant from Biogen-idec. UZ has received personal fees from Biogen Idec GmbH, Bayer Vital GmbH, Bristol Myers Squibb GmbH, CorTec GmbH, Medtronic GmbH, and grants from Biogen Idec GmbH, Servier, and Janssen Pharmaceuticals NV, outside of the submitted work. CSH has received honoraria as editor from Elsevier Publishers, Amsterdam. The remaining authors have no conflicts of interest. 


\section{References}

Alagapan S, Schmidt SL, Lefebvre J, Hadar E, Shin HW, Fröhlich F. Modulation of cortical oscillations by low-frequency direct cortical stimulation is statedependent. PLoS Biol 2016;14:e1002424.

Alekseichuk I, Turi Z, Amador de Lara G, Antal A, Paulus W. Spatial working memory in humans depends on theta and high gamma synchronization in the prefronta cortex. Curr Biol 2016;26:1513-21.

Ali MM, Sellers KK, Fröhlich F. Transcranial alternating current stimulation modulates large-scale cortical network activity by network resonance. J Neurosci 2013;33:11262-75.

Antal A, Boros K, Poreisz C, Chaieb L, Terney D, Paulus W. Comparatively weak aftereffects of transcranial alternating current stimulation (tACS) on cortical excitability in humans. Brain Stimul 2008;1:97-105.

Artola A, Bröcher S, Singer W. Different voltage-dependent thresholds for inducing long-term depression and long-term potentiation in slices of rat visual cortex. Nature 1990;347:69-72.

Barker AT, Jalinous R, Freeston IL. Non-invasive magnetic stimulation of human motor cortex. Lancet 1985;1:1106-7.

Bergmann TO, Groppa S, Seeger M, Mölle M, Marshall L, Siebner HR. Acute changes in motor cortical excitability during slow oscillatory and constant anodal transcranial direct current stimulation. J Neurophysiol 2009;102:2303-11.

Bergmann TO, Mölle M, Schmidt MA, Lindner C, Marshall L, Born J, et al. EEG-guided transcranial magnetic stimulation reveals rapid shifts in motor cortical excitability during the human sleep slow oscillation. J Neurosci 2012a;32:243-53.

Bergmann TO, Mölle M, Diedrichs J, Born J, Siebner HR. Sleep spindle-related reactivation of category-specific cortical regions after learning face-scene associations. Neuroimage 2012b;59:2733-42.

Bergmann TO, Karabanov A, Hartwigsen G, Thielscher A, Siebner HR. Combining non-invasive transcranial brain stimulation with neuroimaging and electrophysiology: current approaches and future perspectives. Neuroimage 2016;140:4-19.

Bestmann S, Feredoes E. Combined neurostimulation and neuroimaging in cognitive neuroscience: past, present, and future. Ann N Y Acad Sci 2013;1296:11-30.

Bestmann S. Computational neurostimulation in basic and translational research. Prog Brain Res 2015;222:xV-X.

Bazhenov M, Timofeev I, Steriade M, Sejnowski TJ. Model of thalamocortical slowwave sleep oscillations and transitions to activated states. J Neurosci 2002;22:8691-704.

Bolognini N, Ro T. Transcranial magnetic stimulation: disrupting neural activity to alter and assess brain function. J Neurosci 2010:30:9647-50.

Bonaiuto JJ, Bestmann S. Understanding the nonlinear physiological and behaviora effects of tDCS through computational neurostimulation. Prog Brain Res 2015;222:75-103.

Bortoletto M, Veniero D, Thut G, Miniussi C. The contribution of TMS-EEC coregistration in the exploration of the human cortical connectome. Neurosc Biobehav Rev 2015;49:114-24.

Bortoletto M, Rodella C, Salvador R, Miranda PC, Miniussi C. Reduced current spread by concentric electrodes in transcranial electrical stimulation (tES). Brain Stimul 2016;9:525-8.

Boyle M., Fröhlich F., 2013. EEG feedback-controlled transcranial alternating current stimulation. 6th Annual International IEEE EMBS Conference on Neural Engineering, pp. 140-3.

Brittain JS, Probert-Smith P, Aziz TZ, Brown P. Tremor suppression by rhythmic transcranial current stimulation. Curr Biol 2013:23:436-40.

Brittain JS, Cagnan H, Mehta AR, Saifee TA, Edwards MJ, Brown P. Distinguishing the central drive to tremor in Parkinson's disease and essential tremor. J Neurosci 2015:35:795-806.

Brunoni AR, Amadera J, Berbel B, Volz MS, Rizzerio BG, Fregni F. A systematic review on reporting and assessment of adverse effects associated with transcrania direct current stimulation. Int J Neuropsychopharmacol 2011;14:1133-45.

Buzsaki G, Draguhn A. Neuronal oscillations in cortical networks. Science 2004;304:1926-9.

Buzsáki G, Anastassiou CA, Koch C. The origin of extracellular fields and currentsEEG, ECoG, LFP and spikes, Nat Rev Neurosci 2012:13:407-20.

Cash RF, Murakami T, Chen R, Thickbroom GW, Ziemann U. Augmenting plasticity induction in human motor cortex by disinhibition stimulation. Cereb Cortex 2016;26:58-69.

Cecere R, Rees G, Romei V. Individual differences in alpha frequency drive crossmodal illusory perception. Curr Biol 2015;25:231-5.

Chander BS, Witkowski M, Braun C, Robinson SE, Born J, Cohen LG, et al. TACS phase locking of frontal midline theta oscillations disrupts working memory performance. Front Cell Neurosci 2016;10:120.

Chanes L, Quentin R, Tallon-Baudry C, Valero-Cabré A. Causal frequency-specific contributions of frontal spatiotemporal patterns induced by non-invasive neurostimulation to human visual performance. I Neurosci 2013;33: 5000-5.

Chanes L, Quentin R, Valero-Cabré A. Arrhythmic activity in the left frontal eye field facilitates conscious visual perception in humans, Cortex 2015:71:240-7.

Debener S, Ullsperger M, Siegel M, Engel AK. Single-trial EEG-fMRI reveals the dynamics of cognitive function. Trends Cogn Sci 2006;10:558-63.

Dugue L, Marque P, Vanrullen R. The phase of ongoing oscillations mediates the causal relation between brain excitation and visual perception. J Neurosci 2011;31:11889-93.
Fertonani A, Miniussi C. Transcranial electrical stimulation: what we know and do not know about mechanisms. Neuroscientist 2016. http://dx.doi.org/10.1177/ 1073858416631966 . Epub ahead of print.

Feurra M, Paulus W, Walsh V, Kanai R. Frequency specific modulation of human somatosensory cortex. Front Psychol 2011;2:13.

Fries P. A mechanism for cognitive dynamics: neuronal communication through neuronal coherence. Trends Cogn Sci 2005;9:474-80.

Fries P. Rhythms for cognition: communication through coherence. Neuron 2015;88:220-35.

Fröhlich F, McCormick DA. Endogenous electric fields may guide neocortical network activity. Neuron 2010;67:129-43.

Fröhlich F. Experiments and models of cortical oscillations as a target for noninvasive brain stimulation. Prog Brain Res 2015;222:41-73.

Fröhlich F, Sellers KK, Cordle AL. Targeting the neurophysiology of cognitive systems with transcranial alternating current stimulation. Expert Rev Neurother 2015;15:145-67.

Goldman DE. Potential impedance, and rectification in membranes. J Gen Physiol $1943 ; 27: 37-60$.

Goldsworthy MR, Vallence AM, Yang R, Pitcher JB, Ridding MC. Combined transcranial alternating current stimulation and continuous theta burst stimulation: a novel approach for neuroplasticity induction. Eur J Neurosci 2016;43:572-9.

Gosseries O, Sarasso S, Casarotto S, Boly M, Schnakers C, Napolitani M, et al. On the cerebral origin of EEG responses to TMS: insights from severe cortical lesions. Brain Stimul 2015;8:142-9.

Groppa S, Bergmann TO, Siems C, Mölle M, Marshall L, Siebner HR. Slow-oscillatory transcranial DC stimulation can induce bidirectional shifts in motor cortical excitability in awake humans. Neuroscience 2010;166:1219-25.

Guerra A, Pogosyan A, Nowak M, Tan H, Ferreri F, Di Lazzaro V, et al. Phase dependency of the human primary motor cortex and cholinergic inhibition cancellation during beta tACS. Cereb Cortex 2016;26:3977-90.

Haegens S, Nácher V, Luna R, Romo R, Jensen O. A-Oscillations in the monkey sensorimotor network influence discrimination performance by rhythmical inhibition of neuronal spiking. Proc Natl Acad Sci USA 2011;108:19377-82.

Hallett M. Transcranial magnetic stimulation: a primer. Neuron 2007;55:187-99.

Hanslmayr S, Matuschek J, Fellner MC. Entrainment of prefrontal beta oscillations induces an endogenous echo and impairs memory formation. Curr Biol 2014;24:904-9.

Hartwigsen G, Bergmann TO, Herz DM, Angstmann S, Karabanov A, Raffin E, et al. Modeling the effects of noninvasive transcranial brain stimulation at the biophysical, network, and cognitive level. Prog Brain Res 2015;222:261-87.

Helfrich RF, Knepper H, Nolte G, Struber D, Rach S, Herrmann CS, et al. Selective modulation of interhemispheric functional connectivity by HD-tACS shapes perception. PLoS Biol 2014a;12:e1002031.

Helfrich RF, Schneider TR, Rach S, Trautmann-Lengsfeld SA, Engel AK, Herrmann CS. Entrainment of brain oscillations by transcranial alternating current stimulation. Curr Biol 2014b;24:333-9.

Herring JD, Thut G, Jensen O, Bergmann TO. Attention modulates TMS-locked alpha oscillations in the visual cortex. J Neurosci 2015;35:14435-47.

Herrmann CS, Rach S, Neuling T, Struber D. Transcranial alternating current stimulation: a review of the underlying mechanisms and modulation of cognitive processes. Front Hum Neurosci 2013;7:279.

Herrmann CS, Murray MM, Ionta S, Hutt A, Lefebvre J. Shaping intrinsic neural oscillations with periodic stimulation. J Neurosci 2016;36:5328-37.

Hill S, Tononi G. Modeling sleep and wakefulness in the thalamocortical system. J Neurophysiol 2005;93:1671-98.

Horvath JC, Forte JD, Carter O. Evidence that transcranial direct current stimulation (tDCS) generates little-to-no reliable neurophysiologic effect beyond MEP amplitude modulation in healthy human subjects: a systematic review. Neuropsychologia 2015;66:213-36.

Huerta PT, Lisman JE. Heightened synaptic plasticity of hippocampal CA1 neurons during a cholinergically induced rhythmic state. Nature 1993;364:723-5.

Huerta PT, Lisman JE. Bidirectional synaptic plasticity induced by a single burst during cholinergic theta oscillation in CA1 in vitro. Neuron 1995:15:1053-63.

Ilmoniemi RJ, Kicic D. Methodology for combined TMS and EEG. Brain Topogr 2010;22:233-48.

Jaegle A, Ro T. Direct control of visual perception with phase-specific modulation of posterior parietal cortex. J Cogn Neurosci 2014;26:422-32.

Jensen O, Colgin LL. Cross-frequency coupling between neuronal oscillations. Trends Cogn Sci 2007;11:267-9.

Jensen O, Mazaheri A. Shaping functional architecture by oscillatory alpha activity: gating by inhibition. Front Hum Neurosci 2010;4:186.

Jensen O, Gips B, Bergmann TO, Bonnefond M. Temporal coding organized by coupled alpha and gamma oscillations prioritize visual processing. Trends Neurosci 2014;37:357-69.

Joundi RA, Jenkinson N, Brittain JS, Aziz TZ, Brown P. Driving oscillatory activity in the human cortex enhances motor performance. Curr Biol 2012;22:403-7.

Karabanov A, Ziemann U, Hamada M, George MS, Quartarone A, Classen J, et al. Consensus paper: probing homeostatic plasticity of human cortex with noninvasive transcranial brain stimulation. Brain Stimul 2015;8:993-1006.

Karabanov A, Thielscher A, Siebner HR. Transcranial brain stimulation: closing the loop between brain and stimulation. Curr Opin Neurol 2016;29:397-404.

Klimesch W, Sauseng P, Gerloff C. Enhancing cognitive performance with repetitive transcranial magnetic stimulation at human individual alpha frequency. Eur J Neurosci 2003;17:1129-33. 
Klooster DC, de Louw AJ, Aldenkamp AP, Besseling RM, Mestrom RM, Carrette S, et al. Technical aspects of neurostimulation: focus on equipment, electric field modeling, and stimulation protocols. Neurosci Biobehav Rev 2016;65:113-41.

Kuo MF, Nitsche MA. Exploring prefrontal cortex functions in healthy humans by transcranial electrical stimulation. Neurosci Bull 2015;31:198-206.

Laakso I, Hirata A. Computational analysis shows why transcranial alternating current stimulation induces retinal phosphenes. J Neural Eng 2013;10:046009.

Lally N, Nord CL, Walsh V, Roiser JP. Does excitatory fronto-extracerebral tDCS lead to improved working memory performance? F1000Res 2013;2:219.

Lee SH, Dan Y. Neuromodulation of brain states. Neuron 2012;76:209-22.

Letzkus JJ, Wolff SB, Luthi A. Disinhibition, a circuit mechanism for associative learning and memory. Neuron 2015;88:264-76.

Li LM, Uehara K, Hanakawa T. The contribution of interindividual factors to variability of response in transcranial direct current stimulation studies. Front Cell Neurosci 2015;9:181.

Liew SL, Santarnecchi E, Buch ER, Cohen LG. Non-invasive brain stimulation in neurorehabilitation: local and distant effects for motor recovery. Front Hum Neurosci 2014;8:378.

Lisman J. The theta/gamma discrete phase code occuring during the hippocampal phase precession may be a more general brain coding scheme. Hippocampus 2005; 15:913-22.

Lorincz ML, Kekesi KA, Juhasz G, Crunelli V, Hughes SW. Temporal framing of thalamic relay-mode firing by phasic inhibition during the alpha rhythm. Neuron 2009;63:683-96.

Lustenberger C, Boyle MR, Alagapan S, Mellin JM, Vaughn BV, Fröhlich F. Feedbackcontrolled transcranial alternating current stimulation reveals a functional role of sleep spindles in motor memory consolidation. Curr Biol 2016;26: 2127-36.

Marshall TR, Esterer S, Herring JD, Bergmann TO, Jensen O. On the relationship between cortical excitability and visual oscillatory responses - a concurrent tDCS-MEG study. Neuroimage 2016;140:41-9.

Martin-Trias P, Bragulat V, Pena-Gomez C, Sala-Llonch R, Lanteaume L, Casse-Perrot $C$, et al. Translational challenge models in support of efficacy studies: neurobehavioral and cognitive changes induced by transcranial magnetic stimulation in healthy volunteers. CNS Neurol Disord Drug Targets 2016;15:802-15.

Mehta AR, Brittain JS, Brown P. The selective influence of rhythmic cortical versus cerebellar transcranial stimulation on human physiological tremor. J Neurosci 2014;34:7501-8.

Mehta AR, Pogosyan A, Brown P, Brittain JS. Montage matters: the influence of transcranial alternating current stimulation on human physiological tremor. Brain Stimul 2015;8:260-8.

Miniussi C, Harris JA, Ruzzoli M. Modelling non-invasive brain stimulation in cognitive neuroscience. Neurosci Biobehav Rev 2013;37:1702-12.

Monto S, Palva S, Voipio J, Palva JM. Very slow EEG fluctuations predict the dynamics of stimulus detection and oscillation amplitudes in humans. J Neurosci 2008:28:8268-72.

Mutanen T, Mäki H, Ilmoniemi RJ. The effect of stimulus parameters on TMS-EEG muscle artifacts. Brain Stimul 2013;6:371-6.

Neuling T, Rach S, Wagner S, Wolters CH, Herrmann CS. Good vibrations: oscillatory phase shapes perception. Neuroimage 2012;63:771-8.

Neuling T, Rach S, Herrmann CS. Orchestrating neuronal networks: sustained aftereffects of transcranial alternating current stimulation depend upon brain states. Front Hum Neurosci 2013;7:161.

Neuling T, Ruhnau P, Fusca M, Demarchi G, Herrmann CS, Weisz N. Friends, not foes: magnetoencephalography as a tool to uncover brain dynamics during transcranial alternating current stimulation. Neuroimage 2015;118:406-13.

Ngo HV, Martinetz T, Born J, Mölle M. Auditory closed-loop stimulation of the sleep slow oscillation enhances memory. Neuron 2013;78:545-53.

Ngo HV, Miedema A, Faude I, Martinetz T, Mölle M, Born J. Driving sleep slow oscillations by auditory closed-loop stimulation-a self-limiting process. J Neurosci 2015;35:6630-8.

Nikouline V, Ruohonen J, Ilmoniemi RJ. The role of the coil click in TMS assessed with simultaneous EEG. Clin Neurophysiol 1999;110:1325-8.

Nitsche MA, Paulus W. Excitability changes induced in the human motor cortex by weak transcranial direct current stimulation. J Physiol 2000;527:633-9.

Noury N, Hipp JF, Siegel M. Physiological processes non-linearly affect electrophysiological recordings during transcranial electric stimulation. Neuroimage 2016;140:99-109.

O'Shea J, Walsh V. Transcranial magnetic stimulation. Curr Biol 2007;17:R196-9.

Opitz A, Paulus W, Will S, Antunes A, Thielscher A. Determinants of the electric field during transcranial direct current stimulation. Neuroimage 2015;109:140-50.

Opitz A, Falchier A, Yan CG, Yeagle EM, Linn GS, Megevand P, et al. Spatiotemporal structure of intracranial electric fields induced by transcranial electric stimulation in humans and nonhuman primates. Sci Rep 2016;6:31236.

Ozen S, Sirota A, Belluscio MA, Anastassiou CA, Stark E, Koch C, et al. Transcranial electric stimulation entrains cortical neuronal populations in rats. J Neurosci 2010;30:11476-85.

Peters JC, Reithler J, Schuhmann T, de Graaf T, Uludag K, Goebel R, et al. On the feasibility of concurrent human TMS-EEG-fMRI measurements. J Neurophysiol 2013;109:1214-27.

Pogosyan A, Gaynor LD, Eusebio A, Brown P. Boosting cortical activity at Beta-band frequencies slows movement in humans. Curr Biol 2009;19:1637-41.

Polania R, Nitsche MA, Korman C, Batsikadze G, Paulus W. The importance of timing in segregated theta phase-coupling for cognitive performance. Curr Biol 2012;22:1314-8.
Prehn K, Flöel A. Potentials and limits to enhance cognitive functions in healthy and pathological aging by tDCS. Front Cell Neurosci 2015;9:355.

Quentin R, Chanes L, Vernet M, Valero-Cabre A. Fronto-parietal anatomical connections influence the modulation of conscious visual perception by high-beta frontal oscillatory activity. Cereb Cortex 2015a;25:2095-101.

Quentin R, Elkin Frankston S, Vernet M, Toba MN, Bartolomeo P, Chanes L, et al Visual contrast sensitivity improvement by right frontal high-beta activity is mediated by contrast gain mechanisms and influenced by fronto-parietal white matter microstructure. Cereb Cortex 2015b;6:2381-90.

Raco V, Bauer R, Tharsan S, Gharabaghi A. Combining TMS and tACS for closed-loop phase-dependent modulation of corticospinal excitability: a feasibility study Front Cell Neurosci 2016:10:143.

Reithler J, Peters JC, Sack AT. Multimodal transcranial magnetic stimulation: using concurrent neuroimaging to reveal the neural network dynamics of noninvasive brain stimulation. Prog Neurobiol 2011;94:149-65.

Rogasch NC, Thomson RH, Farzan F, Fitzgibbon BM, Bailey NW, Hernandez-Pavon JC, et al. Removing artefacts from TMS-EEG recordings using independent component analysis: importance for assessing prefrontal and motor cortex network properties. Neuroimage 2014;101:425-39.

Rogasch NC, Sullivan C, Thomson RH, Rose NS, Bailey NW, Fitzgerald PB, et al Analysing concurrent transcranial magnetic stimulation and electroencephalographic data: a review and introduction to the open-source TESA software. Neuroimage 2016. http://dx.doi.org/10.1016/j. neuroimage.2016.10.031. Epub ahead of print.

Romei V, Brodbeck V, Michel C, Amedi A, Pascual-Leone A, Thut G. Spontaneous fluctuations in posterior alpha-band EEG activity reflect variability in excitability of human visual areas. Cereb Cortex 2008a;18:2010-8.

Romei V, Rihs T, Brodbeck V, Thut G. Resting electroencephalogram alpha-power over posterior sites indexes baseline visual cortex excitability. NeuroReport 2008b;19:203-8.

Romei V, Gross J, Thut G. On the role of prestimulus alpha rhythms over occipitoparietal areas in visual input regulation: correlation or causation? J Neurosci 2010;30:8692-7.

Romei V, Bauer M, Brooks JL, Economides M, Penny W, Thut G, et al. Causal evidence that intrinsic beta-frequency is relevant for enhanced signal propagation in the motor system as shown through rhythmic TMS. Neuroimage 2015;126:120-30.

Romei V, Thut G, Silvanto J. Information-based approaches of noninvasive transcranial brain stimulation. Trends Neurosci 2016;39:782-95.

Rossi S, Hallett M, Rossini PM, Pascual-Leone A. Safety of TMSCG. Safety, ethical considerations, and application guidelines for the use of transcranial magnetic stimulation in clinical practice and research. Clin Neurophysiol 2009;120:2008-39.

Ruhnau P, Neuling T, Fusca M, Herrmann CS, Demarchi G, Weisz N. Eyes wide shut: transcranial alternating current stimulation drives alpha rhythm in a state dependent manner. Sci Rep 2016;6:27138.

Rusu CV, Murakami M, Ziemann U, Triesch J. A model of TMS-induced I-waves in motor cortex. Brain Stimul 2014;7:401-14.

Ruzzoli M, Soto-Faraco S. Alpha stimulation of the human parietal cortex attunes tactile perception to external space. Curr Biol 2014;24:329-32.

Sack AT, Kohler A, Bestmann S, Linden DE, Dechent P, Goebel R, et al. Imaging the brain activity changes underlying impaired visuospatial judgments: simultaneous FMRI, TMS, and behavioral studies. Cereb Cortex 2007; 17:2841-52.

Santarnecchi E, Polizzotto NR, Godone M, Giovannelli F, Feurra M, Matzen L, et al. Frequency-dependent enhancement of fluid intelligence induced by transcranial oscillatory potentials. Curr Biol 2013;23:1449-53.

Sauseng P, Klimesch W, Gerloff C, Hummel FC. Spontaneous locally restricted EEC alpha activity determines cortical excitability in the motor cortex. Neuropsychologia 2009a;47:284-8.

Sauseng P, Klimesch W, Heise KF, Gruber WR, Holz E, Karim AA, et al. Brain oscillatory substrates of visual short-term memory capacity. Curr Biol 2009b;19:1846-52.

Scheeringa R, Petersson KM, Oostenveld R, Norris DG, Hagoort P, Bastiaansen MC Trial-by-trial coupling between EEG and BOLD identifies networks related to alpha and theta EEG power increases during working memory maintenance. Neuroimage 2009;44:1224-38.

Scheeringa R, Mazaheri A, Bojak I, Norris DG, Kleinschmidt A. Modulation of visually evoked cortical FMRI responses by phase of ongoing occipital alpha oscillations. J Neurosci 2011;31:3813-20.

Schmidt S, Iyengar A, Foulser A, Boyle M, Fröhlich F. Endogenous cortical oscillations constrain neuromodulation by weak electric fields. Brain Stimul 2014;7:878-89.

Schmidt S, Bathe-Peters R, Fleischmann R, Ronnefarth M, Scholz M Brandt SA. Nonphysiological factors in navigated TMS studies; confounding covariates and valid intracortical estimates. Hum Brain Mapp 2015;36:40-9.

Schroeder CE, Lakatos P, Kajikawa Y, Partan S, Puce A. Neuronal oscillations and visual amplification of speech. Trends Cogn Sci 2008;12:106-13.

Schroeder CE, Lakatos P. Low-frequency neuronal oscillations as instruments of sensory selection. Trends Neurosci 2009;32:9-18.

Schulz H, Ubelacker T, Keil J, Muller N, Weisz N. Now I am ready-now i am not: The influence of pre-TMS oscillations and corticomuscular coherence on motorevoked potentials. Cereb Cortex 2014;24:1708-19.

Schutter DJ, Hortensius R. Retinal origin of phosphenes to transcranial alternating current stimulation. Clin Neurophysiol 2010;121:1080-4. 
Schutter DJ. Cutaneous retinal activation and neural entrainment in transcranial alternating current stimulation: a systematic review. Neuroimage 2016;140:83-8.

Schwiedrzik CM. Retina or visual cortex? The site of phosphene induction by transcranial alternating current stimulation. Front Integr Neurosci 2009;3:6.

Schyns PG, Thut G, Gross J. Cracking the code of oscillatory activity. PLoS Bio 2011;9:e1001064.

Siebner HR, Peller M, Willoch F, Minoshima S, Boecker H, Auer C, et al. Lasting cortical activation after repetitive TMS of the motor cortex: a glucose metabolic study. Neurology 2000;54:956-63.

Siebner HR, Takano B, Peinemann A, Schwaiger M, Conrad B, Drzezga A. Continuous transcranial magnetic stimulation during positron emission tomography: a suitable tool for imaging regional excitability of the human cortex. Neuroimage 2001;14:883-90.

Siebner HR, Bergmann TO, Bestmann S, Massimini M, Johansen-Berg H, Mochizuki $\mathrm{H}$, et al. Consensus paper: combining transcranial stimulation with neuroimaging. Brain Stimul 2009;2:58-80.

Singer W. Distributed processing and temporal codes in neuronal networks. Cogn Neurodyn 2009;3:189-96.

Sjöström PJ, Turrigiano GG, Nelson SB. Rate, timing, and cooperativity jointly determine cortical synaptic plasticity. Neuron 2001;32:1149-64.

Soekadar SR, Witkowski M, Cossio EG, Birbaumer N, Robinson SE, Cohen LG. In vivo assessment of human brain oscillations during application of transcrania electric currents. Nat Commun 2013a;4:2032.

Soekadar SR, Witkowski M, Robinson SE, Birbaumer N. Combining electric brain stimulation and source-based brain-machine interface (BMI) training in neurorehabilitation of chronic stroke. J Neurol Sci 2013b;333:e542.

Steriade M, Nunez A, Amzica F. A novel slow $(<1 \mathrm{~Hz})$ oscillation of neocortical neurons in vivo: depolarizing and hyperpolarizing components. J Neurosci 1993;13:3252-65.

Steriade M. Grouping of brain rhythms in corticothalamic systems. Neuroscience 2006;137:1087-106.

Thut G, Veniero D, Romei V, Miniussi C, Schyns P, Gross J. Rhythmic TMS causes local entrainment of natural oscillatory signatures. Curr Biol 2011;21:1176-85.

Thut G, Miniussi C, Gross J. The functional importance of rhythmic activity in the brain. Curr Biol 2012;22:R658-63.

Timmermann L, Gross J, Dirks M, Volkmann J, Freund HJ, Schnitzler A. The cerebral oscillatory network of parkinsonian resting tremor. Brain 2003;126:199-212.

Triesch J, Zrenner C, Ziemann U. Modeling TMS-induced I-waves in human motor cortex. Prog Brain Res 2015;222:105-24.

Turi Z, Ambrus GG, Janacsek K, Emmert K, Hahn L, Paulus W, et al. Both the cutaneous sensation and phosphene perception are modulated in a frequencyspecific manner during transcranial alternating current stimulation. Restor Neurol Neurosci 2013;31:275-85.

Utz KS, Dimova V, Oppenländer K, Kerkhoff G. Electrified minds: transcranial direct current stimulation (tDCS) and galvanic vestibular stimulation (GVS) as methods of non-invasive brain stimulation in neuropsychology-a review of current data and future implications. Neuropsychologia 2010;48:2789-810.

VanRullen R, Koch C. Is perception discrete or continuous? Trends Cogn Sci 2003; 7:207-13.

Varela F, Lachaux JP, Rodriguez E, Martinerie J. The brainweb: phase synchronization and large-scale integration. Nat Rev Neurosci 2001;2:229-39.

Veniero D, Bortoletto M, Miniussi C. TMS-EEG co-registration: on TMS-induced artifact. Clin Neurophysiol 2009;120:1392-9.
Veniero D, Vossen A, Gross J, Thut G. Lasting EEG/MEG aftereffects of rhythmic transcranial brain stimulation: level of control over oscillatory network activity. Front Cell Neurosci 2015;9:477.

Vernet M, Thut G. Electroencephalography during transcranial magnetic stimulation: current modus operandi. In: Pascual-Leone A, Horvath J, Rotenberg A, editors. Neuromethods: Transcranial Magnetic Stimulation. Springer; 2014.

Virtanen J, Ruohonen J, Näätänen R, Ilmoniemi RJ. Instrumentation for the measurement of electric brain responses to transcranial magnetic stimulation. Med Biol Eng Comput 1999;37:322-6.

Vossen A, Gross J, Thut G. Alpha power increase after transcranial alternating current stimulation at alpha frequency (alpha-tacs) reflects plastic changes rather than entrainment. Brain Stimul 2015;8:499-508.

Vosskuhl J, Huster RJ, Herrmann CS. BOLD signal effects of transcranial alternating current stimulation (tACS) in the alpha range: a concurrent tACS-fMRI study. Neuroimage 2016. http://dx.doi.org/10.1016/i.neuroimage.2015.10.003. Epub ahead of print.

Wang XJ. Neurophysiological and computational principles of cortical rhythms in cognition. Physiol Rev 2010;90:1195-268.

Wang JX, Rogers LM, Gross EZ, Ryals AJ, Dokucu ME, Brandstatt KL, et al. Targeted enhancement of cortical-hippocampal brain networks and associative memory. Science 2014;345:1054-7.

Wessel MJ, Zimerman M, Hummel FC. Non-invasive brain stimulation: an interventional tool for enhancing behavioral training after stroke. Front Hum Neurosci 2015;9:265.

Witkowski M, Garcia-Cossio EG, Chander BS, Braun C, Birbaumer N, Robinson SE, et al. Mapping entrained brain oscillations during transcranial alternating current stimulation (tACS). Neuroimage 2016;140:89-98.

Womelsdorf T, Everling S. Long-range attention networks: circuit motifs underlying endogenously controlled stimulus selection. Trends Neurosci 2015;38:682-700.

Woods AJ, Antal A, Bikson M, Boggio PS, Brunoni AR, Celnik P, et al. A technical guide to tDCS, and related non-invasive brain stimulation tools. Clin Neurophysiol 2016;127:1031-48

Zaehle T, Rach S, Herrmann CS. Transcranial alternating current stimulation enhances individual alpha activity in human EEG. PLoS One 2010;5:e13766.

Zagha E, McCormick DA. Neural control of brain state. Curr Opin Neurobiol 2014;29:178-86.

Ziemann U. Transcranial magnetic stimulation at the interface with other techniques: a powerful tool for studying the human cortex. Neuroscientist 2011;17:368-81.

Ziemann U, Siebner HR. Inter-subject and inter-session variability of plasticity induction by non-invasive brain stimulation: boon or bane? Brain Stimul 2015;8:662-3.

Zrenner C, Eytan D, Wallach A, Thier P, Marom S. A generic framework for real-time multi-channel neuronal signal analysis, telemetry control, and sub-millisecond latency feedback generation. Front Neurosci 2010:4:173.

Zrenner C, Tünnerhoff J, Zisper C, Müller-Dahlhaus F, Ziemann U. V38. Brain-state dependent non-invasive brain stimulation using closed-loop real-time EEG signal analysis to trigger a TMS pulse with millisecond accuracy. Clin Neurophysiol 2015;126:e85.

Zrenner C, Belardinelli P, Müller-Dahlhaus F, Ziemann U. Closed-loop neuroscience and non-invasive brain stimulation: a tale of two loops. Front Cell Neurosci 2016;10:92. 